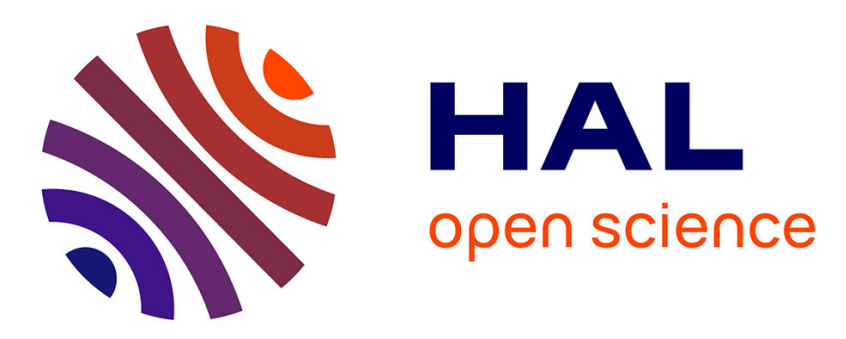

\title{
Earth's spheroidal motion induced by a gravitational wave in flat spacetime
}

\author{
Josipa Majstorović, Séverine Rosat, Yves Rogister
}

\section{To cite this version:}

Josipa Majstorović, Séverine Rosat, Yves Rogister. Earth's spheroidal motion induced by a gravitational wave in flat spacetime. Physical Review D, 2019, 100 (4), 10.1103/PhysRevD.100.044048 . hal-02275862

\section{HAL Id: hal-02275862 \\ https://hal.science/hal-02275862}

Submitted on 19 Jan 2022

HAL is a multi-disciplinary open access archive for the deposit and dissemination of scientific research documents, whether they are published or not. The documents may come from teaching and research institutions in France or abroad, or from public or private research centers.
L'archive ouverte pluridisciplinaire HAL, est destinée au dépôt et à la diffusion de documents scientifiques de niveau recherche, publiés ou non, émanant des établissements d'enseignement et de recherche français ou étrangers, des laboratoires publics ou privés. 


\title{
Earth's spheroidal motion induced by a gravitational wave in flat space-time
}

\author{
Josipa Majstorović, ${ }^{1, \text { * }}$ Séverine Rosat, ${ }^{1}$ and Yves Rogister ${ }^{2}$ \\ ${ }^{1}$ Institut de Physique du Globe de Strasbourg UMR 7516, \\ Université de Strasbourg/EOST, CNRS, \\ 5 rue Descartes, 67000 Strasbourg, France \\ ${ }^{2}$ Université de Strasbourg/EOST, 5 rue Descartes, 67000 Strasbourg, France
}

(Dated: August 1, 2019)

\begin{abstract}
We revisit the theoretical modelling of astrophysical gravitational wave (GW) interaction with Earth's normal modes in the $\mathrm{mHz}$ frequency band. We consider a force term associated with plane waves in a flat space-time acting on a non-rotating anelastic Earth model. Our study confirms that the leading spheroidal induced displacement consists of an angular order-2 term, while the induced azimuthal order terms depend on the incident angles of the GW. We show that some of the previous estimates of the Earth's acceleration due to incoming gravitational waves were too optimistic. Current ground-based detectors are embedded in seismic and environmental noise that is several orders of magnitude larger than the expected GW signal. However, with the prospect of new technologies and improved noise reduction techniques, detection may become feasible.
\end{abstract}

Keywords: Gravitational wave detection, Earth's interior, Gravitational wave source, Gravitational waves

\footnotetext{
*jmajstorovic@unistra.fr
} 


\section{INTRODUCTION}

On September 14, 2015 at 09:50:45 UTC for the first time in history the two detectors of the Laser Interferometer Gravitational-Wave Observatory (LIGO) simultaneously detected gravitational wave $(\mathrm{GW})$ from binary hole merger [1]. It was an additional indisputable proof of the theory of general relativity published by Albert Einstein in 1916, who found transverse waves that travel at the speed of light as a solution for the weak-field equation of his theory. These waves are generated by the time variation of the mass quadrupole moment of the sources [2, 3]. The detection consisted of a transient GW signal with the peak strain of $1.1 \times 10^{-21}$ whose frequency increased with time (chirp) from 35 to $250 \mathrm{~Hz}$ in $0.15 \mathrm{~s}$. The signal matched the predicted waveform for the inspiral and merger of a pair of black holes and the ringdown of the resulting signal black hole. After the main detection ten more followed, thus until today there are eleven successfully identified mergers, ten of stellar-mass binary black hole mergers and one binary neutron star [4]. Currently The Advanced LIGO [5] and The Advanced Virgo [6] are in the third observation run, known as O3, which began 1st of April, 2019 and is scheduled for one calendar year. The updates on detectors statuses and GW Candidate Event Database can be followed on the website https://www.gw-openscience.org/about/.

The detections were obtained with the Advanced LIGO and the Advanced VIRGO, the interferometric ground-based detectors, which have been operating in observational mode during the event. The Advanced LIGO has a wide detection range from $10 \mathrm{~Hz}$ to $7000 \mathrm{~Hz}$, with the most sensitive band from $100 \mathrm{~Hz}$ to $300 \mathrm{~Hz}$ [1, 5]. In addition to the ground-based laser interferometers or resonant bars, which are high-frequency observations sensitive in $\mathrm{kHz}$ band $\left(\sim 1\right.$ to $\sim 10^{4} \mathrm{~Hz}$ ), the space-based detectors will be developed (e.g. eLISA [7]) with the sensitivity in $\mathrm{mHz}$ band constructed for the strain deformation measurement. Complementary to these low-frequency detectors is also Doppler tracking of spacecraft. Further, the pulsar timing arrays (PTAs) [8], very-low frequency observations sensitive in

the band $\sim 10^{-8}$ to $\sim 10^{-5} \mathrm{~Hz}$, are constructed for the measurement of the arrival time from the milliseconds pulsars. And finally, the extremely low frequency observations in the band $\sim 10^{-18}$ to $\sim 10^{-15} \mathrm{~Hz}$ are focused on the cosmic microwave background measurements of primordial origin. GW signals are roughly divided into three categories: those from well determined sources, stochastic backgrounds and unmodelled transient sources. All 
sources together cover a wide range of frequencies, however not all of these sources produce a gravitational strain detectable on Earth. The highest characteristic strain is induced by the stochastic sources in the frequency range from $10^{-10}$ to $10^{-6} \mathrm{~Hz}$ and it decreases as the frequency increases [9].

Around the same time when the first idea about the ground-based detector was established, the idea that GWs could excite the vibrations of elastic bodies, and therefore Earth too, was developed [10, 11]. Weber [11] proposed methods for the interstellar gravitational radiation detection using the fact that the relative motion of mass points are driven by second spatial derivatives of the gravitational fields. He proposed an experiment where the Earth is considered as a block of material representing the GW antenna, a resonant body, for which the normal modes of the Earth are expected as a response to the excitation. He also discussed generation and detection of GW in the laboratory. Forward et. al. [12] were the first to calculate the upper bound of the GW energy passing through the Earth using the strain data from the seismograph at Isabella, California. They computed the strain magnitude induced by the Riemann tensor in a longitudinally vibrating rod [11]. Next, Weber [13] provided the first upper limit on the gravitational-radiation flux using a mechanical gravimeter in vicinity of normal mode periods. Tuman [14, 15] first claimed a GW detection using the Earth's normal modes observed in cryogenic gravity meter records. He interpreted a higher energy content in the power spectra of the even harmonic degrees as the normal modes harmonics excited by gravitational radiation. His finding was criticized due to a lack of more convincing statistical proof [16]. An important study was done by Dyson [17] who was the first to calculate the response of a flat-Earth model to an incident GW, where effects of sphericity and rotation were added to the flat stationary Earth solution. The calculated response was in the $1-\mathrm{Hz}$ band where seismic wavelengths are small compared to the Earth's radius and large compared to lateral density heterogeneities. Dyson [17] showed that the GWs, in such a set-up, are absorbed only by irregularities in the shear-modulus, with the strongest absorption at free surface. Sabbata et. al. [18] proposed detecting GWs by the observation of Earth's free oscillations. Their apparatus consisted of laser interferometer which allows to measure the soil deformations. They proposed that distinction of the seismic (free oscillations) from the gravitational signals could be accomplished by considering a long interval of time, to look at the Fourier components at the presumed frequencies and consider the decay time of the oscillations. Mast et. al. [19] performed the search 
for gravitational radiation from pulsars using a seismometer on the Earth. Even though no signal was found, they estimated an upper limit on the Earth motion due to such signal from $10^{-11} \mathrm{~m}$ near $1 \mathrm{~Hz}$ to $10^{-14} \mathrm{~m}$ near $125 \mathrm{~Hz}$. Extensive work on the reception of GW by an elastic self-gravitating spherical detector was done by Ashby et. al. [20]. The equations of motion of a detector are presented in the coordinate system of Fermi, where the GW field appears as a classical driving force, and exact analytic solutions are modeled for the homogeneous isotropic elastic sphere as well as self-stress sphere, where stress on the body due to its own gravitational field causes radial variations in density and elastic moduli in equilibrium state. The elastic response was calculated for monochromatic waves in the range $10^{-4} \mathrm{~Hz}$ to $1 \mathrm{~Hz}$. Similar work was done by Linet [21], where he modeled the equations governing the interaction between non-rotating elastic self-gravitating sphere and GWs. Based on [17], Jensen [22] analysed the absorption of GW in the 1-Hz band by the layered crust of a realistic Earth model, developing the interaction between GWs and the elastic continuum. Jensen [22] showed that discontinuities in the elastic modulus in a layered model significantly enhance the response of Earth to GWs at specific frequencies. The complete response of the radially heterogeneous rotating and self-gravitating Earth in terms of induced toroidal and spheroidal motions was then developed by Ben-Menahem [23]. He showed that in the long-wavelength regime for the induced spheroidal vibration the most significant response corresponds to quadrupole modes. More recently, Coughlin and Harms [24-26] revisited Dyson's and Ben-Menahem's formalism of the Earth response to incident GW for the calculation of the upper limit of GW energy density. In the first paper [24] they used a global network of broadband seismometers and they considered isotropic stochastic GW background integrated over one year in the frequency range 0.05-1 Hz. In the second paper [25] they used data from a superconducting gravimeter network in the frequency range 0.035-0.15 Hz. In the third paper [26] they used Apollo-era seismic data integrated over one year in the frequency range 0.1-1 Hz.

Besides Weber and Dyson, who were pioneers in considering the Earth as a detector of GW, many papers that followed studied the interaction of $\mathrm{GW}$ and elastic solids in the general relativity context. One of the first studies modeled a concept of the perfectly elastic solid in the high-pressure elasticity theory (condition that occurs in the interiors of neutron stars) for the purpose of scrutinizing the interaction of gravitational radiation with planetary bodies [27]. Also, for the fist time the strain-curvature equation for an elastic test 
body interacting with a GW was formulated in general relativistic systems [28]. Further, the interaction problem was also solved in the gravito-inertial system of reference [29, 30]. In the later paper [30], it was emphasized the existence of the superposition of two different elastic waves, those with the phase velocity equal to the speed of light and those with the phase velocity equal to the seismic velocity.

The first two papers to consider absorption of GW by astrophysical objects were Zimmerman and Hellings [31] and Walgate [32]. Boughn and Kuhn [33] were the first one to use realistic Earth and Sun models to calculate their responses to the homogeneous isotropic gravitational radiation considering coupling of GW to a spherically symmetric body. Thus, they put upper limits on the stochastic gravitational background from the observed solar oscillations. Khosroshahi and Sobouti [34] studied the excitation possibility of the polytropic stars normal modes. They showed that the interaction is achieved through the irrotational component of the displacement vector field. Siegel and Roth [35-38] published four articles on the topic of the non-relativistic stars excitation by arbitrary external GW fields starting from the full field equations of general relativity. In [35], it was shown that GWs solely couple to quadrupole spheroidal eigenmodes. In their next paper [36], they developed a hydrodynamic model of the excited normal modes for any non-relativistic star and arbitrary external GW fields, allowing them to use realistic current solar and stellar models. They studied two types of radiation, either from a particular astrophysical source or from a stochastic background. In the third and fourth papers [37, 38, based on their earlier theoretical work, they showed that asteroseismology can place upper bounds on the amplitude of a stochastic background of GWs in the $\mathrm{mHz}$ and $\mu \mathrm{Hz}$ frequency range. Recently, Lopes and Silk [39 41] and Lopes [42] published studies on the stars quadrupole acoustic modes. They showed that GWs with a strain spectral amplitude in the range $10^{-20}-10^{-17}$ can lead to the excitation of Sun's low order quadrupole acoustic modes [39]. They also argued that stars in general form a natural set of detectors over a large spectral frequency range, from $10^{-7}$ to $10^{-2} \mathrm{~Hz}$, and that their stellar configuration is ideal for GW search. Unlike experimental detectors this kind of network of stars allow to study the progression of GWs throughout space [40]. Lopes [42] calculated that impact of GWs on low-order quadrupole modes is not above the current observational threshold of detectability, however he concluded it may be reached with the next generation of near infrared observatories and asteroseismology satellite missions. Among others, the studies about the absorption of GW by stars near black 
holes and white-dwarfs [43] and red giant stars [44] also exist. A historical summary of these papers is presented in the chart in Fig. 1.

Earth

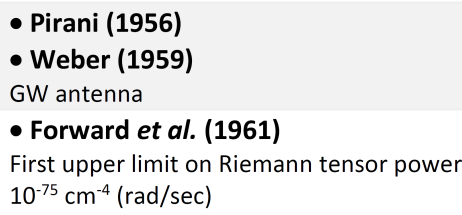

- Weber (1967)

Upper limit on $\rho_{p}(\omega)<2 \cdot 10^{-25} \mathrm{~g} \mathrm{~cm}^{-3} \mathrm{rad}^{-1} \mathrm{sec}$

- Dyson (1969)

Flat model, $f=1 \mathrm{~Hz}, u_{h}=2 \cdot 10^{-17} \mathrm{~m}$

- Sabbata et. al. (1970)

- Tuman (1971)

First claimed detection using free oscillations

- Tuman (1973)

- Mast et. al. (1974)

$u=10^{-11} \mathrm{~m}(1 \mathrm{~Hz})-10^{-14} \mathrm{~m}(125 \mathrm{~Hz})$

- Ashby et. al. (1975)

Spherical self-gravitating model, $f=10^{-4}-1 \mathrm{~Hz}$

- Jensen (1979)

Layered/crustal model, $f=0.1-10 \mathrm{~Hz}, u=5 \cdot 10^{-20} \mathrm{~m}$

- Ben-Menahem (1983)

Radially Heterogeneous Rotating SG, $f=<10 \mathrm{mHz}$,

$u_{h}=10^{-9} \mathrm{~m}$

\section{General spherical bodies}

Astrophysical

bodies

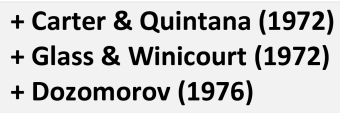

+ Linet (1984)

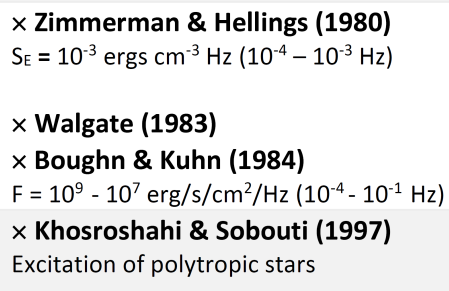

FIG. 1. Historical chart representing the progress of the utilization of Earth as a detector of GWs $(\bullet)$. Additionally, the studies utilizing general elastic sphere $(+)$ and astrophysical bodies $(\times)$ are also shown. Abbreviations stands for: $\rho_{P}(\omega)$ - power spectrum of the gravitational-radiation mass density, $f$ - frequency/frequency range, $u$ - displacement, $u_{h}$ - horizontal displacement, $\Omega_{\mathrm{GW}}$ upper limit on energy density, $S_{E}$ - spectral energy density, $F$ - flux density. 
The main objective of this paper is to revisit and upgrade Ben-Menahem's [23] equations and expressions of the spheroidal motions induced by GW using a different formalism. Particularly, we use different seismological formalism and gravitational source definition. The paper is organized as follows. Firstly, in the theoretical section we review the GW in a flat space-time and we introduce the definition of gravitational force upon an elastic body. We review the formalism for non-rotating anelastic Earth model deformation and we derive the induced spheroidal motion by GW. Secondly, using derived equations we predict the induced displacement and we present the state of art of the published detection thresholds. Finally, we discuss how detection using Earth resonance may be improved in the future.

\section{THEORY}

\section{A. Gravitational waves in flat space-time}

Far away from any significant masses, space-time possesses no curvature. It is the flat space-time approximation, where we can idealize the waves as plane-fronted. The appropriate formalism for describing this approximation is a linearized theory of gravity, a consequence of considering the Newtonian limit (moving from a curved space-time to a flat one). In the Newtonian limit particles are moving slowly with respect to the speed of light, the gravitational field is weak and so it can be considered as a perturbation of flat space and the field is static. The weakness of the gravitational field is expressed as decomposition of the metric into the Minkowski metric plus a small perturbation

$$
g_{\mu \nu}=\eta_{\mu \nu}+h_{\mu \nu}, \quad\left|h_{\mu \nu}\right| \ll 1
$$

where the metric tensor $g_{\mu \nu}$ is a function of the distribution of mass and energy in space and time, $\eta_{\mu \nu}$ takes its canonical form $\eta_{\mu \nu}=\operatorname{diag}(-1,1,1,1)$ and $h_{\mu \nu}$ is the metric perturbation. The assumption $\left|h_{\mu \nu}\right| \ll 1$ allows us to ignore anything that is higher order than first order in metric perturbation. In this assumption metric perturbation can be chosen so as to satisfy a transverse-traceless gauge condition where $h_{\mu 0}=0$ is purely spatial, $h_{m m}=0$ trace free and $h_{m n ; n}=0$ divergence free. In this gauge all components $h_{\mu \nu}$ obey the wave equation where the solutions are plane waves, of which phase fronts are huge compared to the wavelength and the radius of curvature of the space-time through which they propagate. We express 
perturbation of the metric as (further we are dropping Einstein summation convention)

$$
\mathbf{h}=\Re\left\{h_{0} \epsilon \mathrm{e}^{i\left(\omega_{g} t-\mathbf{k}_{g} \cdot \mathbf{r}\right)}\right\},
$$

where $\Re$ designates the real part, $h_{0}$ defines the intensity of the wave source, $\boldsymbol{\epsilon}$ is a polarization tensor, $\mathbf{k}_{g}=\frac{\omega_{g}}{c} \hat{\mathbf{e}}_{k}$ is the wavenumber with $\omega_{g}$ as frequency, $c$ the velocity of light and $\hat{\mathbf{e}}_{k}$ a unit vector normal to the wave front of the GW. The polarization tensor is defined by the configuration of the incoming $\mathrm{GW}$ propagating along a vector $\mathbf{k}_{g}$, which points from the source to the observer, located in the Earth reference system. To define this tensor the direction of propagation $\mathbf{k}_{g}$ is not sufficient, we also need to chose a vector $\mathbf{l}$ perpendicular to $\mathbf{k}_{g}$, and a third vector $\mathbf{m}=\mathbf{k}_{g} \times \mathbf{l}$ to specify the basis vectors for the tensor construction. Vectors $\mathbf{k}_{g}, \mathbf{l}, \mathbf{m}$ are determined in the Earth's Cartesian coordinate system $\mathcal{O}$, with the $\hat{\mathbf{e}}_{z}$-axis pointing toward North, the $\hat{\mathbf{e}}_{x}$ and $\hat{\mathbf{e}}_{y}$-axes perpendicular to $\hat{\mathbf{e}}_{z}$, and whose origin coincides with the center of mass of the Earth. Unit vector $\hat{\mathbf{e}}_{x}$ points to the Greenwich meridian. The plane of polarization in the $\mathcal{O}$-system is specified by three angles $\{e, \lambda, \nu\}$, where $e$ defines the rotation in $\hat{\mathbf{e}}_{y} \hat{\mathbf{e}}_{z}$-plane, $\lambda$ in $\hat{\mathbf{e}}_{x} \hat{\mathbf{e}}_{y}$-plane and $\nu$ is the rotation angle about the unit vector $\hat{\mathbf{e}}_{k}$ (Fig. 2).

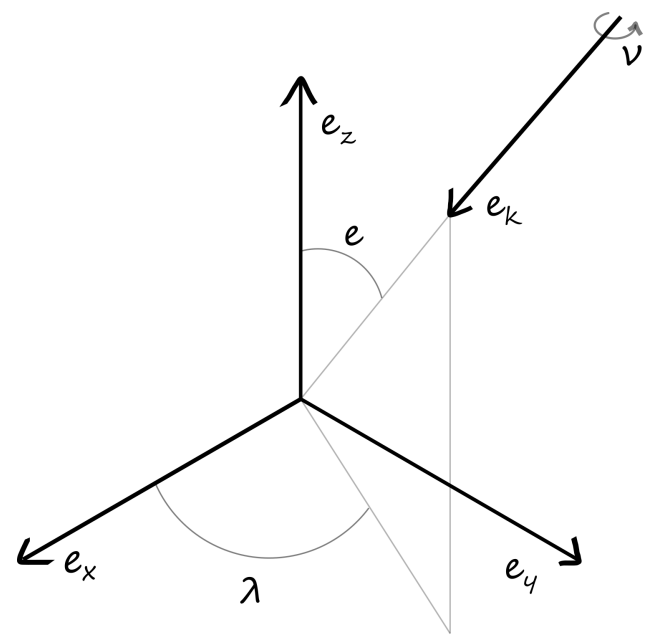

FIG. 2. Cartesian coordinate system $\mathcal{O}$, where its origin coincides with the center of mass of the Earth and $\hat{\mathbf{e}}_{z}$-axis points to the North, $\hat{\mathbf{e}}_{x}$-axis toward the Greenwich Meridian and $\hat{\mathbf{e}}_{y}$-axis is perpendicular to the $\hat{\mathbf{e}}_{z} \hat{\mathbf{e}}_{x}$-plane. 
Thus in the system $\mathcal{O}$ we have

$$
\begin{aligned}
\hat{\mathbf{e}}_{e} & =(\cos e \cos \lambda, \cos e \sin \lambda,-\sin e), \\
\hat{\mathbf{e}}_{\lambda} & =(-\sin \lambda, \cos \lambda, 0), \\
\hat{\mathbf{e}}_{k} & =(\sin e \cos \lambda, \sin e \sin \lambda, \cos e) .
\end{aligned}
$$

Further, rotation about GW-propagation axis gives us the unit vectors

$$
\begin{aligned}
\mathbf{l} & =\cos \nu\left(-\hat{\mathbf{e}}_{e}\right)+\sin \nu\left(-\hat{\mathbf{e}}_{\lambda}\right), \\
\mathbf{m} & =\sin \nu\left(\hat{\mathbf{e}}_{e}\right)+\cos \nu\left(-\hat{\mathbf{e}}_{\lambda}\right) .
\end{aligned}
$$

From the GW formalism we are familiar with the two polarization tensors $\mathbf{e}_{+}$, $\mathbf{e}_{\times}$which can be defined using unit vectors (4) as

$$
\begin{aligned}
& \mathbf{e}_{+}=\mathbf{l} \otimes \mathbf{l}-\mathbf{m} \otimes \mathbf{m}, \\
& \mathbf{e}_{\times}=\mathbf{l} \otimes \mathbf{m}+\mathbf{m} \otimes \mathbf{l} .
\end{aligned}
$$

Considering the linearly polarized GW, tensor $\boldsymbol{\epsilon}$ is defined as

$$
\boldsymbol{\epsilon}=(\mathbf{l} \otimes \mathbf{l}-\mathbf{m} \otimes \mathbf{m})+(\mathbf{l} \otimes \mathbf{m}+\mathbf{m} \otimes \mathbf{l}) .
$$

whence, in terms of angles $\{e, \lambda, \nu\}$, we have (with the shorthand $\sin =s$ and $\cos =c$ )

$$
\boldsymbol{\epsilon}=\left[\begin{array}{ccc}
b_{1}\left(c^{2}(e) c^{2}(\lambda)-s^{2}(\lambda)\right)-b_{2} c(e) s(2 \lambda) & \frac{1}{2} b_{1} s(2 \lambda)\left(c^{2}(e)+1\right)+b_{2} c(e) c(2 \lambda) & -\frac{1}{2} b_{1} s(2 e) c(\lambda)+b_{2} s(e) s(\lambda) \\
\frac{1}{2} b_{1} s(2 \lambda)\left(c^{2}(e)+1\right)+b_{2} c(e) c(2 \lambda) & b_{1}\left(c^{2}(e) s^{2}(\lambda)-c^{2}(\lambda)\right)+b_{2} c(e) s(2 \lambda) & -\frac{1}{2} b_{1} s(2 e) s(\lambda)-b_{2} s(e) c(\lambda) \\
-\frac{1}{2} b_{1} s(2 e) c(\lambda)+b_{2} s(e) s(\lambda) & -\frac{1}{2} b_{1} s(2 e) s(\lambda)-b_{2} s(e) c(\lambda) & b_{1} s^{2}(e)
\end{array}\right],
$$

with

$$
\begin{aligned}
& b_{1}=\cos 2 \nu-\sin 2 \nu, \\
& b_{2}=\cos 2 \nu+\sin 2 \nu .
\end{aligned}
$$

When considering a weak field linear approximation, Dyson [17] showed that in the interior of an isotropic elastic medium a GW interacts only with the discontinuities in the shear modulus profile $\mu$, which gives rise to the force term [17, 23]

$$
\mathbf{f}(\mathbf{r}, t)=-\frac{\partial \mu}{\partial r} \hat{\mathbf{e}}_{r} \cdot \mathbf{h}=-h_{0} \frac{\partial \mu}{\partial r} \hat{\mathbf{e}}_{r} \cdot \boldsymbol{\epsilon} \mathrm{e}^{i\left(\omega_{g} t-\mathbf{k}_{g} \cdot \mathbf{r}\right)},
$$

where $\mu$ is the shear modulus distribution in the Earth and $\hat{\mathbf{e}}_{r}$ is the radial unit vector. When considering a realistic Earth model there are two major discontinuities in the shear-modulus profile: at the free-surface and at the core-mantle boundary. To define the response of the Earth to the GW we need to consider the force term (9) as a body force in the relevant linear elastic equations of motion. 


\section{B. Green tensor formalism}

The Earth's response to any source which excites free oscillations or traveling body and surface waves can be expressed in terms of the second-order Green tensor $\mathbf{G}\left(\mathbf{r}, \mathbf{r}^{\prime} ; t\right)$. It represents a displacement response at location $\mathbf{r}$ and time $t$ to a force acting at location $\mathbf{r}^{\prime}$ at time 0 . Therefore, the displacement $\mathbf{s}(\mathbf{r}, t)$ produced by the equivalent body force density $\mathbf{f}(\mathbf{r}, t)$ acting per unit volume and the equivalent surface force density $\mathbf{t}(\mathbf{r}, t)$ can be written as a convolution of the impulse response Green function $\mathbf{G}\left(\mathbf{r}, \mathbf{r}^{\prime} ; t\right)$ with the entire past history of the equivalent forces $\mathbf{f}(\mathbf{r}, t)$ and $\mathbf{t}(\mathbf{r}, t)$ as 45$]$

$$
\mathbf{s}(\mathbf{r}, t)=\int_{-\infty}^{t} \int_{V} \mathbf{G}\left(\mathbf{r}, \mathbf{r}^{\prime} ; t-t^{\prime}\right) \cdot \mathbf{f}\left(\mathbf{r}^{\prime}, t^{\prime}\right) d V^{\prime} d t^{\prime}+\int_{-\infty}^{t} \int_{S} \mathbf{G}\left(\mathbf{r}, \mathbf{r}^{\prime} ; t-t^{\prime}\right) \cdot \mathbf{t}\left(\mathbf{r}^{\prime}, t^{\prime}\right) d \Sigma^{\prime} d t^{\prime}
$$

where the volume integral is carried throughout the Earth's volume and the surface integral over Earth's surface satisfying a dynamical free-surface boundary condition

$$
\hat{\mathbf{n}} \cdot \mathbf{T}=0
$$

for all surface. $\hat{\mathbf{n}}$ is vector normal to the surface and $\mathbf{T}$ is the elastic stress tensor

$$
\mathbf{T}=\Gamma: \varepsilon,
$$

where $\boldsymbol{\Gamma}$ is a fourth-order elastic tensor and $\varepsilon$ stress tensor. Taking into account that the elastic wave speed is much smaller than the speed of light, we consider that the whole Earth is simultaneously excited, therefore the effect of rotation is ignored [46]. The Green tensor for a non-rotating, anelastic Earth, represented as a sum of seismic normal modes with associated complex eigenfrequencies $\nu_{k}=\omega_{k}+i \gamma_{k}=\omega_{k}+i \frac{\omega_{k}}{2 Q_{k}}$, is written as

$$
\mathbf{G}\left(\mathbf{r}, \mathbf{r}^{\prime} ; t\right)=\Re \sum_{k}\left(i \nu_{k}\right)^{-1} \mathbf{s}_{k}(\mathbf{r}) \mathbf{s}_{k}\left(\mathbf{r}^{\prime}\right) \mathrm{e}^{i \nu_{k} t} .
$$

The associated displacement eigenfunction $\mathbf{s}_{k}$ is written in the form of real spherical harmonics

$$
\mathbf{s}_{k}(\mathbf{r})=\mathcal{U}_{k}(r) \hat{\mathbf{e}}_{r} \mathcal{Y}_{l m}(\theta, \phi)+\kappa^{-1} \mathcal{V}_{k}(r) \nabla_{1} \mathcal{Y}_{l m}(\theta, \phi)-\kappa^{-1} \mathcal{W}_{k}(r)\left(\hat{\mathbf{e}}_{r} \times \nabla_{1} \mathcal{Y}_{l m}(\theta, \phi)\right)
$$

The real spherical harmonics are defined as

$$
\mathcal{Y}_{l m}(\theta, \phi)=\left\{\begin{array}{l}
\sqrt{2} X_{l|m|}(\theta) \cos m \phi \text { if }-l \leq m<0 \\
X_{l 0}(\theta) \text { if } m=0 \\
\sqrt{2} X_{l m}(\theta) \sin m \phi \text { if } 0<m \leq l
\end{array}\right.
$$


with

$$
X_{l m}=(-1)^{m} \sqrt{\frac{2 l+1}{4 \pi}} \sqrt{\frac{(l-m) !}{(l+m) !}} P_{l m}(\cos \theta) .
$$

$P_{l m}(\cos \theta)$ is the associated Legendre function defined as

$$
P_{l m}(x)=\frac{(-1)^{m}}{2^{l} l !}\left(1-x^{2}\right)^{m / 2} \frac{d^{l+m}}{d x^{l+m}}\left(x^{2}-1\right)^{l}
$$

with $x=\cos \theta$. Radial scalar functions $\mathcal{U}_{k}(r), \mathcal{V}_{k}(r), \mathcal{W}_{k}(r)$ are complex, $\nabla_{1}=\hat{\mathbf{e}}_{\theta} \partial_{\theta}+$ $\hat{\mathbf{e}}_{\phi}(\sin \theta)^{-1} \partial_{\phi}$ is the surface gradient and $\kappa=\sqrt{l(l+1)}$. For a spherically symmetric Earth, the index $k$ denotes the quadruplet $\{n, l, m, \mathrm{~S}$ or $\mathrm{T}\}$, where $n$ is radial overtone number, $l$ is angular order and $m$ azimuthal order of spherical harmonic and $\mathrm{S}$ and $\mathrm{T}$, stand for spheroidal and toroidal modes, respectively. Toroidal displacements have $\mathcal{U}_{k}=0, \mathcal{V}_{k}=0$ in (14), whereas spheroidal ones have $\mathcal{W}_{k}=0$. For practical reasons in the calculation of synthetic seismograms on a spherical Earth, only the effect of anelasticity upon the eigenfrequencies is retained and the effect of the anelasticity upon the radial eigenfunctions is ignored, so (14) becomes

$$
\mathbf{s}_{k}(\mathbf{r})=U_{k}(r) \hat{\mathbf{e}}_{r} \mathcal{Y}_{l m}(\theta, \phi)+\kappa^{-1} V_{k}(r) \nabla_{1} \mathcal{Y}_{l m}(\theta, \phi)-\kappa^{-1} W_{k}(r)\left(\hat{\mathbf{e}}_{r} \times \nabla_{1} \mathcal{Y}_{l m}(\theta, \phi)\right),
$$

where $U_{k}(r), V_{k}(r), W_{k}(r)$ are real functions.

\section{Induced spheroidal motion}

To obtain the solution for the spheroidal motion induced by a GW defined by the force term (9), we need to insert this term into Green tensor (10) and take into account the boundary condition (11) on the surface. Using the weak field linear approximation, the interaction between GW and an elastic solid can be taken into account by adding a term [17, 23 .

$$
\mathbf{T}^{G W}=-\mu \mathbf{h}
$$

into the stress tensor, which results in the gravitational tidal force in the equation of motion, shown elsewhere [20, 21]. Therefore, the boundary condition (11) is altered and the surface force density becomes

$$
\mathbf{t}=\mu(a) \hat{\mathbf{e}}_{r} \cdot \mathbf{h} .
$$


where $\mu(a)$ is the value of the shear modulus at the Earth's surface. Considering the above relation, equation 10 becomes

$$
\begin{aligned}
\mathbf{s}(\mathbf{r}, t)= & -\int_{-\infty}^{t} \int_{V} \frac{\partial \mu}{\partial r} \mathbf{G}\left(\mathbf{r}, \mathbf{r}^{\prime} ; t-t^{\prime}\right) \cdot\left(\hat{\mathbf{e}}_{r} \cdot \mathbf{h}\right) d V^{\prime} d t^{\prime} \\
& +\mu(a) \int_{-\infty}^{t} \int_{S} \mathbf{G}\left(\mathbf{r}, \mathbf{r}^{\prime} ; t-t^{\prime}\right) \cdot\left(\hat{\mathbf{e}}_{r} \cdot \mathbf{h}\right) d \Sigma^{\prime} d t^{\prime} .
\end{aligned}
$$

and substituting (2) and (13) we have for a given seismic mode $k$

$$
\begin{aligned}
\mathbf{s}_{k}(\mathbf{r}, t)= & -h_{0} \mathbf{s}_{k}(\mathbf{r}) \bar{g}(t) \int_{V} \frac{\partial \mu}{\partial r} \mathbf{s}_{k}\left(\mathbf{r}^{\prime}\right) \cdot\left(\hat{\mathbf{e}}_{r} \cdot \boldsymbol{\epsilon} \mathrm{e}^{-i \mathbf{k}_{g} \cdot \mathbf{r}^{\prime}}\right) d V^{\prime} \\
& +\mu(a) h_{0} \mathbf{s}_{k}(\mathbf{r}) \bar{g}(t) \int_{S} \mathbf{s}_{k}\left(\mathbf{r}^{\prime}\right) \cdot\left(\hat{\mathbf{e}}_{r} \cdot \boldsymbol{\epsilon} \mathrm{e}^{-i \mathbf{k}_{g} \cdot \mathbf{a}^{\prime}}\right) d \Sigma^{\prime}
\end{aligned}
$$

where we have extracted the source-time function

$$
\bar{g}(t)=\int_{-\infty}^{t}\left(i \nu_{k}\right)^{-1} \mathrm{e}^{i \nu_{k}\left(t-t^{\prime}\right)} \mathrm{e}^{i \omega_{g} t^{\prime}} d t^{\prime} .
$$

Convolution in the time domain is equivalent to multiplication in the frequency domain, that is $\mathcal{F}\{f(t) * g(t)\}=\mathcal{F}\{f(t)\} \cdot \mathcal{F}\{g(t)\}$, where $\mathcal{F}$ is Fourier transform. Using this theorem we obtain

$$
\begin{aligned}
\bar{g}(t) & =\frac{1}{2 \pi} \int_{-\infty}^{+\infty}\left(i \nu_{k}\right)^{-1} \mathcal{F}\{f(t)\} \cdot \mathcal{F}\{g(t)\} \mathrm{e}^{i \omega t} d \omega \\
& =\frac{1}{2 \pi} \int_{-\infty}^{+\infty}\left(i \nu_{k}\right)^{-1} \delta\left(\omega-\omega_{g}\right) \frac{1}{\gamma_{k}+i\left(\omega-\omega_{k}\right)} \mathrm{e}^{i \omega t} d \omega \\
& =\frac{1}{2 \pi}\left(i \nu_{k}\right)^{-1} \frac{1}{\gamma_{k}+i\left(\omega_{g}-\omega_{k}\right)} \mathrm{e}^{i \omega_{g} t} .
\end{aligned}
$$

In the long-wavelength regime we expect $k_{g} a \ll 1[20,21$, 34], thus we simplify (22) into

$$
\begin{aligned}
\mathbf{s}_{k}(\mathbf{r}, t) & =h_{0} \mathbf{s}_{k}(\mathbf{r}) \bar{g}(t)\left[-\int_{V} \frac{\partial \mu}{\partial r} \mathbf{s}_{k}\left(\mathbf{r}^{\prime}\right) \cdot\left(\hat{\mathbf{e}}_{r} \cdot \boldsymbol{\epsilon}\right) d V^{\prime}+\mu(a) \int_{S} \mathbf{s}_{k}\left(\mathbf{r}^{\prime}\right) \cdot\left(\hat{\mathbf{e}}_{r} \cdot \boldsymbol{\epsilon}\right) d \Sigma^{\prime}\right] \\
& =h_{0} \mathbf{s}_{k}(\mathbf{r}) \bar{g}(t) \boldsymbol{\epsilon}:\left[-\int_{V} \frac{\partial \mu}{\partial r} \mathbf{s}_{k}\left(\mathbf{r}^{\prime}\right) \hat{\mathbf{e}}_{r} d V^{\prime}+\mu(a) \int_{S} \mathbf{s}_{k}\left(\mathbf{r}^{\prime}\right) \hat{\mathbf{e}}_{r} d \Sigma^{\prime}\right] .
\end{aligned}
$$

Substituting the definition of the displacement eigenfunction (18) for the spheroidal modes $(W=0)$ we obtain

$$
\begin{aligned}
\mathbf{s}_{k}(\mathbf{r}, t)=h_{0} \mathbf{s}_{k}(\mathbf{r}) \bar{g}(t) \boldsymbol{\epsilon}: & {\left[\left(\mu(a) U_{k}(a) a^{2}-\int_{r} \frac{\partial \mu}{\partial r} U_{k}(r) r^{2} d r\right) \int_{\Omega} \hat{\mathbf{e}}_{r} \hat{\mathbf{e}}_{r} \mathcal{Y}_{l m}(\theta, \phi) d \Omega\right.} \\
+ & \left.\left(\mu(a) \kappa^{-1} V_{k}(a) a^{2}-\int_{r} \frac{\partial \mu}{\partial r} \kappa^{-1} V_{k}(r) r^{2} d r\right) \int_{\Omega} \hat{\mathbf{e}}_{r} \nabla_{1} \mathcal{Y}_{l m}(\theta, \phi) d \Omega\right]
\end{aligned}
$$


where $d \Omega=\sin \theta d \theta d \phi$ with $0<\phi<2 \pi, 0<\theta<\pi$ and $\int_{r}$ is integral over radius from center $(r=0)$ to surface $(r=a)$. To solve integrals in 26$)$ we need to define the unit vectors $\left\{\hat{\mathbf{e}}_{r}, \hat{\mathbf{e}}_{\theta}, \hat{\mathbf{e}}_{\phi}\right\}$ and their dyadic products $\left\{\hat{\mathbf{e}}_{r} \hat{\mathbf{e}}_{r}, \hat{\mathbf{e}}_{r} \hat{\mathbf{e}}_{\theta}, \hat{\mathbf{e}}_{r} \hat{\mathbf{e}}_{\phi}\right\}$. For this we use the expressions of the unit vectors $\left\{\hat{\mathbf{e}}_{r}, \hat{\mathbf{e}}_{\theta}, \hat{\mathbf{e}}_{\phi}\right\}$ in spherical coordinates

$$
\left[\begin{array}{c}
\hat{\mathbf{e}}_{r} \\
\hat{\mathbf{e}}_{\theta} \\
\hat{\mathbf{e}}_{\phi}
\end{array}\right]=\left[\begin{array}{ccc}
\sin \theta \cos \phi & \sin \theta \sin \phi & \cos \theta \\
\cos \theta \cos \phi & \cos \theta \sin \phi & -\sin \theta \\
-\sin \theta & \cos \phi & 0
\end{array}\right]\left[\begin{array}{c}
\hat{\mathbf{e}}_{x} \\
\hat{\mathbf{e}}_{y} \\
\hat{\mathbf{e}}_{z}
\end{array}\right]
$$

and calculate dyadic products using definition $\mathbf{a b}=\sum_{j=1}^{3} \sum_{i=1}^{3} a_{i} b_{j} \hat{\mathbf{e}}_{i} \hat{\mathbf{e}}_{j}$. The first integral in $(26)$

$$
I_{1}=\int_{\Omega} \hat{\mathbf{e}}_{r} \hat{\mathbf{e}}_{r} \mathcal{Y}_{l m}(\theta, \phi) d \Omega=\int_{0}^{\pi} \int_{0}^{2 \pi} \hat{\mathbf{e}}_{r} \hat{\mathbf{e}}_{r} \mathcal{Y}_{l m}(\theta, \phi) \sin \theta d \theta d \phi
$$

is a double integral over two arguments $\phi$ and $\theta$ over nine components of the dyadic product $\left\{\hat{\mathbf{e}}_{r} \hat{\mathbf{e}}_{r}\right\}$. The solution is

$I_{1}=\frac{2 \sqrt{\pi}}{3} \delta_{l, 0} \delta_{m, 0}\left[\begin{array}{lll}1 & 0 & 0 \\ 0 & 1 & 0 \\ 0 & 0 & 1\end{array}\right]+\frac{2}{3} \sqrt{\frac{\pi}{5}} \delta_{l, 2} \delta_{m, 0}\left[\begin{array}{ccc}-1 & 0 & 0 \\ 0 & -1 & 0 \\ 0 & 0 & 2\end{array}\right]+2 \sqrt{\frac{\pi}{15}} \delta_{l, 2}\left[\begin{array}{ccc}\delta_{m,-2} & \delta_{m, 2} & -\delta_{m,-1} \\ \delta_{m, 2} & -\delta_{m,-2} & -\delta_{m, 1} \\ -\delta_{m,-1} & -\delta_{m, 1} & 0\end{array}\right]$,

where we define the Kronecker delta symbol as

$$
\delta_{i, j}=\left\{\begin{array}{l}
0 \text { if } i \neq j \\
1 \text { if } i=j
\end{array} .\right.
$$

Next, we derive the second integral in relation (26), which is

$$
\begin{aligned}
I_{2}=\int_{\Omega} \hat{\mathbf{e}}_{r} \nabla_{1} \mathcal{Y}_{l m}(\theta, \phi) d \Omega & =\int_{0}^{\pi} \int_{0}^{2 \pi} \hat{\mathbf{e}}_{r} \hat{\mathbf{e}}_{\theta} \partial_{\theta} \mathcal{Y}_{l m}(\theta, \phi) \sin \theta d \theta d \phi \\
& +\int_{0}^{\pi} \int_{0}^{2 \pi} \hat{\mathbf{e}}_{r} \hat{\mathbf{e}}_{\phi}(\sin \theta)^{-1} \partial_{\phi} \mathcal{Y}_{l m}(\theta, \phi) \sin \theta d \theta d \phi .
\end{aligned}
$$

The two integrals in (31) also involve nine integrals due to $\left\{\hat{\mathbf{e}}_{r} \hat{\mathbf{e}}_{\theta}\right\}$ and $\left\{\hat{\mathbf{e}}_{r} \hat{\mathbf{e}}_{\phi}\right\}$ dyadic products and the final result is

$$
I_{2}=2 \sqrt{\frac{\pi}{5}} \delta_{l, 2} \delta_{m, 0}\left[\begin{array}{ccc}
-1 & 0 & 0 \\
0 & -1 & 0 \\
0 & 0 & 2
\end{array}\right]+6 \sqrt{\frac{\pi}{15}} \delta_{l, 2}\left[\begin{array}{ccc}
\delta_{m,-2} & \delta_{m, 2} & -\delta_{m,-1} \\
\delta_{m, 2} & -\delta_{m,-2} & -\delta_{m, 1} \\
-\delta_{m,-1} & -\delta_{m, 1} & 0
\end{array}\right]
$$


Finally, the expression (26) becomes

$$
\begin{aligned}
\mathbf{s}_{k}(\mathbf{r}, t)=h_{0} \mathbf{s}_{k}(\mathbf{r}) \bar{g}(t) \boldsymbol{\epsilon}: & \left\{\frac{2 \sqrt{\pi}}{3} \delta_{l, 0} \delta_{m, 0}\left[\begin{array}{lll}
1 & 0 & 0 \\
0 & 1 & 0 \\
0 & 0 & 1
\end{array}\right]\left(\mu(a) U_{k}(a) a^{2}-\int_{r} \frac{\partial \mu}{\partial r} U_{k}(r) r^{2} d r\right)\right. \\
+ & \left(\frac{2}{3} \sqrt{\frac{\pi}{5}} \delta_{l, 2} \delta_{m, 0}\left[\begin{array}{ccc}
-1 & 0 & 0 \\
0 & -1 & 0 \\
0 & 0 & 2
\end{array}\right]+2 \sqrt{\frac{\pi}{15}} \delta_{l, 2}\left[\begin{array}{ccc}
\delta_{m,-2} & \delta_{m, 2} & -\delta_{m,-1} \\
\delta_{m, 2} & -\delta_{m,-2} & -\delta_{m, 1} \\
-\delta_{m,-1} & -\delta_{m, 1} & 0
\end{array}\right]\right) \\
& \left.\left(\mu(a) a^{2}\left(U_{k}(a)+\frac{3}{\sqrt{6}} V_{k}(a)\right)-\int_{r} \frac{\partial \mu}{\partial r}\left(U_{k}(r)+\frac{3}{\sqrt{6}} V_{k}(r)\right) r^{2} d r\right)\right\} .
\end{aligned}
$$

However since $\boldsymbol{\epsilon}: \mathrm{I}=0$ ( $\mathrm{I}$ is the identity matrix) the above expression reduces to

$$
\begin{gathered}
\mathbf{s}_{k}(\mathbf{r}, t)=h_{0} \mathbf{s}_{k}(\mathbf{r}) \bar{g}(t) \delta_{l, 2} \boldsymbol{\epsilon}:\left\{\frac{2}{3} \sqrt{\frac{\pi}{5}} \delta_{m, 0}\left[\begin{array}{ccc}
-1 & 0 & 0 \\
0 & -1 & 0 \\
0 & 0 & 2
\end{array}\right]+2 \sqrt{\frac{\pi}{15}}\left[\begin{array}{ccc}
\delta_{m,-2} & \delta_{m, 2} & -\delta_{m,-1} \\
\delta_{m, 2} & -\delta_{m,-2} & -\delta_{m, 1} \\
-\delta_{m,-1} & -\delta_{m, 1} & 0
\end{array}\right]\right\} \\
\left(\mu(a) a^{2}\left(U_{k}(a)+\frac{3}{\sqrt{6}} V_{k}(a)\right)-\int_{r} \frac{\partial \mu}{\partial r}\left(U_{k}(r)+\frac{3}{\sqrt{6}} V_{k}(r)\right) r^{2} d r\right) .
\end{gathered}
$$

The complete contraction between $\boldsymbol{\epsilon}$ and matrices appearing in (34) yields the expression

$$
\mathbf{s}_{k}(\mathbf{r}, t)=h_{0} \mathbf{s}_{k}(\mathbf{r}) \bar{g}(t) \delta_{l, 2} f^{m}(e, \lambda, \nu) \alpha_{k},
$$

with

$$
\alpha_{k}=\mu(a) a^{2}\left(U_{k}(a)+\frac{3}{\sqrt{6}} V_{k}(a)\right)-\int_{r} \frac{\partial \mu}{\partial r}\left(U_{k}(r)+\frac{3}{\sqrt{6}} V_{k}(r)\right) r^{2} d r
$$

and the function $f^{m}(e, \lambda, \nu)$ defined as

$$
\begin{aligned}
f^{m}(e, \lambda, \nu) & =\frac{2}{3} \sqrt{\frac{\pi}{5}} \delta_{m, 0} b_{1} \sin ^{2} e \\
& +\delta_{m, 2} \frac{C}{2}\left[4 b_{2} \cos e \cos 2 \lambda+b_{1}(3+\cos 2 e) \sin 2 \lambda\right] \\
& +\delta_{m,-2} C\left[b_{1} \cos 2 \lambda\left(\cos ^{2} e+1\right)-2 b_{2} \cos e \sin 2 \lambda\right] \\
& +\delta_{m, 1} 2 C \sin e\left[b_{2} \cos \lambda+b_{1} \cos e \sin \lambda\right] \\
& -\delta_{m,-1} 2 C \sin e\left[b_{2} \sin \lambda-b_{1} \cos e \cos \lambda\right]
\end{aligned}
$$

with $C=2 \sqrt{\frac{\pi}{15}}$. According to 35 the leading angular term for the induced spheroidal motion is $\delta_{l, 2}$ and therefore associated azimuth terms are $\{m=-2,-1,0,1,2\}$ [23, 33 35, 47]. This result comes from the fact that in general relativity $h_{\mu \nu}$ is traceless and 
symmetric [47]. The displacement depends on the value of the scalar $h_{0}$ which is defined by the gravitational source, the displacement eigenfunction $\mathbf{s}_{k}$, the source-time function $\bar{g}(t)$, the function $f^{m}(e, \lambda, \nu)$ defining the incoming GW and a constant $\alpha_{k}$ which depends on the Earth model. The three components of the displacement (35) at $\mathbf{r}$ are

$$
\begin{gathered}
{ }_{n} s_{2, r}^{m}=h_{0}{ }_{n} \alpha_{2}{ }_{n} U_{2} \mathcal{Y}_{l m}(\theta, \phi) f^{m}(e, \lambda, \nu) \Re\{\bar{g}(t)\}, \\
{ }_{n} s_{2, \theta}^{m}=h_{0} \alpha_{2} \frac{1}{\sqrt{6}}{ }_{n} V_{2} \partial_{\theta} \mathcal{Y}_{l m}(\theta, \phi) f^{m}(e, \lambda, \nu) \Re\{\bar{g}(t)\}, \\
{ }_{n} s_{2, \phi}^{m}=h_{0}{ }_{n} \alpha_{2} \frac{1}{\sqrt{6}}{ }_{n} V_{2}(\sin \theta)^{-1} \partial_{\phi} \mathcal{Y}_{l m}(\theta, \phi) f^{m}(e, \lambda, \nu) \Re\{\bar{g}(t)\},
\end{gathered}
$$

with

$$
\Re\{\bar{g}(t)\}=\frac{1}{2 \pi} \frac{\left(\omega_{k}^{2}-\omega_{k} \omega_{g}-\gamma_{k}^{2}\right) \cos \omega_{g} t-\left(\gamma_{k} \omega_{g}-2 \gamma_{k} \omega_{k}\right) \sin \omega_{g} t}{\left(\omega_{k}^{2}+\gamma_{k}^{2}\right)\left[\gamma_{k}^{2}+\left(\omega_{g}^{2}-\omega_{k}^{2}\right)\right]} .
$$

We see that the incident angles of the GW $\{e, \lambda, \mu\}$ determine which normal modes are

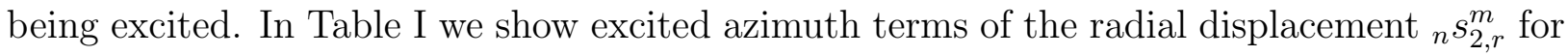
different combinations of $\{e, \lambda, \mu\}$ angles.

TABLE I. Excited degree-2 azimuthal terms for different combinations of the $\{e, \lambda, \mu\}$ angles defining the incoming gravitational wave.

\begin{tabular}{lc}
\hline \hline$e, \lambda, \mu\}$ & $m$ \\
\hline$\{0,0,0\},\left\{0, \frac{\pi}{2}, 0\right\},\left\{0,0, \frac{\pi}{2}\right\},\left\{0, \frac{\pi}{2}, \frac{\pi}{2}\right\}$ & $-2,2$ \\
$\left\{\frac{\pi}{2}, 0,0\right\},\left\{\frac{\pi}{2}, 0, \frac{\pi}{2}\right\}$ & $-2,1,0$ \\
$\left\{\frac{\pi}{2}, 0,0\right\},\left\{\frac{\pi}{2}, \frac{\pi}{2}, 0\right\},\left\{\frac{\pi}{2}, \frac{\pi}{2}, \frac{\pi}{2}\right\}$ & $-2,-1,0$ \\
\hline \hline
\end{tabular}

We emphasise that we are primarily interested in the Earth oscillatory motion continuously forced by a GW. We neglect the impulse reponse of the Earth to the initial excitation by the GW. We also neglect the transient motion that follows the initial excitation and decays before the oscillatory regime is reached.

\section{DISCUSSION}

The relations (38)-(40) are essentially the same as relations (47)-(49) in 23. However, differences exist since we used a different definition of spherical harmonics, different polarization of GW and Green tensor formalism from [45]. Next, we derive an expression for the 
right-hand circularly polarized GW, defined as $\frac{1}{2}\left(\mathbf{e}_{+}-i \mathbf{e}_{\times}\right)$, since this one was used in [23]. Therefore, the polarization tensor is defined as

$$
\boldsymbol{\epsilon}_{b}=\frac{1}{2} \mathrm{e}^{2 i \nu}\left[\begin{array}{ccc}
(c(e) c(\lambda)+i s(\lambda))^{2} & -i c(e) c(2 \lambda)+\frac{1}{2}\left(1+c^{2}(e)\right) s(2 \lambda) & -s(e)(c(e) c(\lambda)+i s(\lambda)) \\
-i c(e) c(2 \lambda)+\frac{1}{2}\left(1+c^{2}(e)\right) s(2 \lambda) & -(c(\lambda)+i c(e) s(\lambda))^{2} & i s(e)(c(\lambda)+i c(e) s(\lambda)) \\
-s(e)(c(e) c(\lambda)+i s(\lambda)) & i s(e)(c(\lambda)+i c(e) s(\lambda)) & s^{2}(e)
\end{array}\right],
$$

and corresponds to the polarization tensor of equation (15) in [23]. For the newly defined tensor the function $f^{m}(e, \lambda, \nu)$ in the displacement vector (35) becomes

$$
\begin{aligned}
f_{b}^{m}(e, \lambda, \nu) & =\sqrt{\frac{\pi}{5}} \delta_{m, 0} \mathrm{e}^{2 i \nu} \sin ^{2} e \\
& +\delta_{m,-1} C \mathrm{e}^{2 i \nu} \sin e[\cos e \cos \lambda+i \sin \lambda] \\
& +\delta_{m, 1} C \mathrm{e}^{2 i \nu} \sin e[\cos e \sin \lambda-i \cos \lambda] \\
& +\delta_{m,-2} \frac{C}{4} \mathrm{e}^{2 i \nu}[3 \cos 2 \lambda+\cos 2 e \cos 2 \lambda+4 i \cos e \sin 2 \lambda] \\
& +\delta_{m, 2} \frac{C}{4} \mathrm{e}^{2 i \nu}[3 \sin 2 \lambda+\cos 2 e \sin 2 \lambda-4 i \cos e \cos 2 \lambda]
\end{aligned}
$$

with $C=2 \sqrt{\frac{\pi}{15}}$ as in Eq. 37 . The values in Table I are valid for the relation 43 too.

Considering this new expression the radial component of the induced spheroidal quadrupole response can be written as

$$
{ }_{n} s_{2, r}^{m}=h_{0{ }_{n}} \alpha_{2}{ }_{n} U_{2} \mathcal{Y}_{l m}(\theta, \phi) \Re\left\{f_{b}^{m}(e, \lambda, \nu) \bar{g}(t)\right\} .
$$

This expression is compared directly with the equivalent relation (47) from [23] which is

$$
\begin{aligned}
& { }_{n} u_{r}=(-1)^{m} \frac{2}{\sqrt{6}} h_{0} \omega_{2}^{2} \bar{g}_{n}(t)\left[y_{1 n}(r) \frac{\mu(a) a^{2}\left(y_{1 n}+3 y_{3 n}\right)-\int_{0}^{a} \frac{\partial \mu}{\partial r}\left(y_{1 n}(r)+3 y_{3 n}(r)\right) r^{2} d r}{{ }_{n} \omega_{2}^{2} \int_{0}^{a}\left(y_{1 n}^{2}(r)+6 y_{3 n}^{2}(r)\right) r^{2} d r}\right] \\
& \Re\left\{\mathrm{e}^{2 i \nu} P_{2 m}(\cos \theta) \sin ^{2-m}\left(\frac{e}{2}\right) \cos ^{2+m}\left(\frac{e}{2}\right) \mathrm{e}^{i m(\phi+\lambda)}\right\} .
\end{aligned}
$$

We rewrite the radial induced response (45) in terms that are comparable to relation (44):

$$
{ }_{n} u_{r}=h_{0}{ }_{n} \alpha_{2}^{\mathrm{BM} 83} y_{1 n}(r) Y_{l m}(\theta, \phi) \Re\left\{f_{b}^{m ; \mathrm{BM} 83}(e, \lambda, \nu) \bar{g}(t)\right\},
$$

where

$$
{ }_{n} \alpha_{2}^{\mathrm{BM} 83}=\frac{\mu(a) a^{2}\left(y_{1 n}+3 y_{3 n}\right)-\int_{0}^{a} \frac{\partial \mu}{\partial r}\left(y_{1 n}(r)+3 y_{3 n}(r)\right) r^{2} d r}{\int_{0}^{a}\left(y_{1 n}^{2}(r)+6 y_{3 n}^{2}(r)\right) r^{2} d r},
$$


and

$$
\begin{aligned}
f_{b}^{m ; \mathrm{BM} 83}(e, \lambda, \nu) & =\delta_{m, 0} \frac{1}{2 \sqrt{6}} \mathrm{e}^{2 i \nu} \sin ^{2} e \\
& +\delta_{m, 2} \frac{2}{\sqrt{6}} \mathrm{e}^{2 i \nu} \cos ^{4}\left(\frac{e}{2}\right) \mathrm{e}^{2 i m} \\
& +\delta_{m,-2} \frac{2}{\sqrt{6}} \mathrm{e}^{2 i \nu} \sin ^{4}\left(\frac{e}{2}\right) \mathrm{e}^{-2 i m} \\
& -\delta_{m, 1} \frac{2}{\sqrt{6}} \mathrm{e}^{2 i \nu} \sin \left(\frac{e}{2}\right) \cos ^{3}\left(\frac{e}{2}\right) \mathrm{e}^{i m} \\
& -\delta_{m,-1} \frac{2}{\sqrt{6}} \mathrm{e}^{2 i \nu} \sin ^{3}\left(\frac{e}{2}\right) \cos \left(\frac{e}{2}\right) \mathrm{e}^{-i m}
\end{aligned}
$$

Therefore, by directly comparing (44) and (46), we see there are several differences. At the beginning, it should be emphasised that in these two relations the notations for the displacement eigenfunction are different, thus we have $U_{k}(r)=y_{1 n}(r)$ and $V_{k}(r)=y_{3 n}(r)$. Thus, the first difference concerns the model dependent functions (48) and (36), which are dissimilar due to the different normalization used for the displacement eigenfunctions that actually depends on the spherical harmonics normalization. In [45] one uses the orthonormalized spherical harmonics and in [23] the Schmidt semi-normalized spherical harmonics. The radial eigenfunctions in [45] are orthonormal due to the general orthonormality of displacement eigenfunctions (see page 279 in [45]). This is not the case for the formalism in [48] and the reader is referred to page 379 for further details. The second difference concerns the used definition of the spherical harmonics. Beside a different normalization for the spherical harmonics, we use real spherical harmonics. This essentially affects the results of the two integrals (29) and (32). The integrals additionally generate a third difference and these are dissimilarities between functions (43) and (48), a consequence of the contraction between the polarization tensor and integrals outputs. For the reader interested on how to transform one formalism into another and how to treat these two formalism, we refer to the Appendix.

To further compare the solution revisited in this study with [23], we will estimate the values of three components of the displacement from 38 to 40 with the function $f^{m}(e, \lambda, \nu)$ defined as 43. We focus on the ${ }_{0} \mathrm{~S}_{2}$ normal mode and the monochromatic source at the resonance frequency $\omega_{g}=\omega_{k}$ with the sensor position at the equator, i.e. at $\left\{\theta=\frac{\pi}{2}, \phi=\frac{\pi}{2}\right\}$, and source angles $\left\{e=\frac{\pi}{2}, \lambda=0, \nu=0\right\}$, which gives

$$
f_{b}^{m}(e, \lambda, \nu)=\sqrt{\frac{\pi}{5}} \delta_{m, 0}-i 2 \sqrt{\frac{\pi}{15}} \delta_{m, 1}+\sqrt{\frac{\pi}{15}} \delta_{m,-2}
$$

The eigenfunctions and eigenfrequencies for a spherically symmetric, non-rotating Earth 
model are calculated for a transversely isotropic PREM model [49], modified for the oceanless case, using MINEOS software package [50]. These calculations are later used for obtaining the constant value $\alpha_{k}$ given by (36). Further, we consider that an idealized accelerometer responds to the perturbation in gravitational potential and free-air change in the gravity in addition to the particle acceleration. These corrections are accounted for by replacing the PREM eigenfuntions $U_{k}, V_{k}$ by $\bar{U}_{k}=U_{k}+\omega_{k}^{-2} 2 a^{-1} g U_{k}+(l+1) \omega_{k}^{-2} a^{-1} P_{k}$ and $\bar{V}_{k}=$ $V_{k}-\kappa \omega_{k}^{-2} a^{-1} g U_{k}-\kappa \omega^{-2} a^{-1} P_{k}$, where $P_{k}$ is the gravitational potential [20, 25, 33, 45] and $g$ the gravity at the surface. Thus, we have

$$
\begin{aligned}
& { }_{0} \bar{U}_{2}={ }_{0} U_{2}+\left({ }_{0} \omega_{2}\right)^{-2} 2 a^{-1} g{ }_{0} U_{2}+3\left({ }_{0} \omega_{2}\right)^{-2} a^{-1}{ }_{0} P_{2} \\
& { }_{0} \bar{V}_{2}={ }_{0} V_{2}-\sqrt{6}\left({ }_{0} \omega_{2}\right)^{-2} a^{-1} g{ }_{0} U_{2}-\sqrt{6}\left({ }_{0} \omega_{2}\right)^{-2} a^{-1}{ }_{0} P_{2} \\
& { }_{0} s_{2, r}=\sum_{m}{ }_{0} s_{2, r}{ }^{m}=h_{00} \alpha_{2}{ }_{0} \bar{U}_{2} \sum_{m} \mathcal{Y}_{2 m}(\theta, \phi) \Re\left\{f_{b}^{m}(e, \lambda, \nu) \bar{g}(t)\right\} \\
& =-\frac{1}{4 \pi} h_{0}{ }_{0} \alpha_{2}{ }_{0} \bar{U}_{2} \frac{{ }_{0} \omega_{2} \sin _{0} \omega_{2} t-{ }_{0} \gamma_{2} \cos { }_{0} \omega_{2} t}{{ }_{0} \gamma_{2}\left({ }_{0} \omega_{2}{ }^{2}+{ }_{0} \gamma_{2}{ }^{2}\right)}, \\
& { }_{0} s_{2, \theta}=\sum_{m}{ }_{0} s_{2, \theta}{ }^{m}=-\frac{1}{\pi 2 \sqrt{6}} h_{0}{ }_{0} \alpha_{2}{ }_{0} \bar{V}_{2} \frac{0 \gamma_{2} \sin _{0} \omega_{2} t+{ }_{0} \omega_{2} \cos { }_{0} \omega_{2} t}{{ }_{0} \gamma_{2}\left({ }_{0} \omega_{2}{ }^{2}+{ }_{0} \gamma_{2}{ }^{2}\right)} \text {, } \\
& { }_{0} s_{2, \phi}=\sum_{m}{ }_{0} s_{2, \phi}{ }^{m}=0 .
\end{aligned}
$$

Using the values from Table II we calculate the radial and tangential displacements (52) and (53) depending on the GW source amplitude $h_{0}$ and for $t=0$. The values are then multiplied by the radius of the Earth $(a=6371 \mathrm{~km})$ to account for the normalization and by $10^{9}$ to convert from meters to nanometers, thus we have

$$
\begin{aligned}
& { }_{0} s_{2, r} \approx 6.9 \cdot 10^{13} h_{0} \mathrm{~nm} \approx 6.9 \cdot 10^{-8} \mathrm{~nm}, \\
& { }_{0} s_{2, \theta} \approx 2.5 \cdot 10^{16} h_{0} \mathrm{~nm} \approx 2.5 \cdot 10^{-5} \mathrm{~nm} .
\end{aligned}
$$

Further, we can calculate the acceleration by multiplying by ${ }_{0} \omega_{2}^{2}$

$$
\begin{gathered}
{ }_{0} a_{2, r} \approx 2.6 \cdot 10^{8} h_{0} \mathrm{~nm} / \mathrm{s}^{2} \approx 2.6 \cdot 10^{-13} \mathrm{~nm} / \mathrm{s}^{2} \\
{ }_{0} a_{2, \theta} \approx 9.6 \cdot 10^{10} h_{0} \mathrm{~nm} / \mathrm{s}^{2} \approx 9.6 \cdot 10^{-11} \mathrm{~nm} / \mathrm{s}^{2}
\end{gathered}
$$

where we set $h_{0}=10^{-21}$ for the representation purpose only, since this was the strain value obtained at the recent first observation of GW [1]. However, in the $\mathrm{mHz}$ frequency band we expect to have different strain values for the binary black hole mergers. 
TABLE II. MINEOS normalized values of the eigenfunctions $U, V, P$ at the Earth surface $r=a$, frequency $\omega$, quality factor $Q$ for ${ }_{0} \mathrm{~S}_{2}$ and gravity value at the surface $g$. Used normalization for length is $R=6371 \mathrm{~km}$, time $\frac{1}{\pi G \rho_{a}}$ and mass $\rho_{a} R^{3}$, where $G=6.67408 \cdot 10^{-11} \mathrm{~m}^{3} \mathrm{~kg}^{-1} \mathrm{~s}^{-2}$ is the gravitational constant and $\rho_{a}=5515.0 \mathrm{kgm}^{-3}$ is the average density.

\begin{tabular}{llllllll}
\hline \hline$U(a)$ & $V(a)$ & $P(a)$ & $\alpha$ & $\omega$ & $\mathrm{Q}$ & $\mathrm{g}$ & $\mathrm{a}$ \\
\hline 1.329 & 0.030 & -0.847 & 0.273 & 1.807 & 509.648 & 1.333 & 1 \\
\hline \hline
\end{tabular}

For the same mode, Ben-Menahem's calculations in [23] consisted of the same set of source angles, but with the sensor position at $\{\theta=0, \phi=0\}$. Further, for the resonance and $h_{0}=10^{-21}$ he found that the values for the horizontal displacement may reach the level of ${ }_{0} s_{2, \theta, 0} s_{2, \phi} \approx 10^{-8} \mathrm{~cm}=0.1 \mathrm{~nm}$, which gives ${ }_{0} a_{2, \theta, 0} a_{2, \phi} \approx 3.6 \cdot 10^{-7} \mathrm{~nm} / \mathrm{s}^{2}$, four orders of magnitude bigger than estimated in this study. However, this result should not be directly compared to our estimate because we do not use the same source type and therefore not the same source-time function. Ben-Menahem used a finite monochromatic wave source whereas we use an infinite monochromatic wave source. Only by considering a different source-time function there is already a difference of four orders of magnitude. Additionally, our sourcetime function definition for an infinite monochromatic source definition differs from the one in [23], because our definition of the Green tensors differs. We also checked Ben-Menahem's calculations by using his equations and approximations. For an infinite monochromatic source we obtain ${ }_{0} u_{\theta}=1.6 \cdot 10^{-6} \mathrm{~nm}$ and ${ }_{0} a_{\theta}=1.4 \cdot 10^{-13} \mathrm{~nm} / \mathrm{s}^{2}$, one order of magnitude smaller than our estimate. We believe that this order of magnitude difference still comes from a different definition of the source-time functions. Also, a significant difference is that in [23] calculations are done for Jeffreys-Bullen and Gutenberg-Bullard Earth models [51], whereas we use the PREM model.

When one talks about the signal detection at the Earth surface one should consider two factors, the first one is the instrument precision and stability and the other is the environmental noise level [52]. Only by combining all these information together with the possible detection threshold we can resolve the estimation of elusive signals, like GW signals. The minimal envelope of the environmental seismic noise may be represented by the widely used New Low Noise Model (NLNM) [53]. This model was developed empirically by taking the lowest noise levels recorded on the $\approx 10$-day-long vertical components at 75 stations, 
after all earthquakes and transients were removed. The NLNM corresponds then to the lower envelope of power spectral densities (PSDs) calculated for all available seismometers. Since the NLNM is flat in the $\mathrm{mHz}$ band a rough estimate of the noise standard deviation at ${ }_{0} \mathrm{~S}_{2}$ frequency is $\sigma_{N L N M}=8.0538 \cdot 10^{-9} \mathrm{~m} / \mathrm{s}^{2}(-151.88 \mathrm{~dB})$. With the power spectral density (PSD) of the white noise defined as $\mathrm{PSD}_{\text {noise }}=\sigma^{2} T_{0}$ (where $\sigma$ is noise standard deviation and $T_{0}$ is sampling interval) and the PSD of undamped harmonic signal defined as $\mathrm{PSD}_{\text {signal }}=\frac{A^{2} N T_{0}}{4}$ (where $N$ is number of data points, $A$ is amplitude of the signal), our signal amplitude needs to satisfy the relation $A>\frac{2 \sigma}{\sqrt{N}}$ to be visible in the noisy data. Thus, to detect the radial component (56) buried in the noise at the level defined above, one would need $5 \cdot 10^{23}$ years, which is completely unfeasible. One can look at this differently and say that for this particular noise standard deviation and 10 years of recorded data with $T_{0}=10$ $\mathrm{s}$, one would need the GW amplitude signal to be larger than $h_{0} \approx 10^{-10}$ to be detected on Earth, which is unrealistically large [9]. 


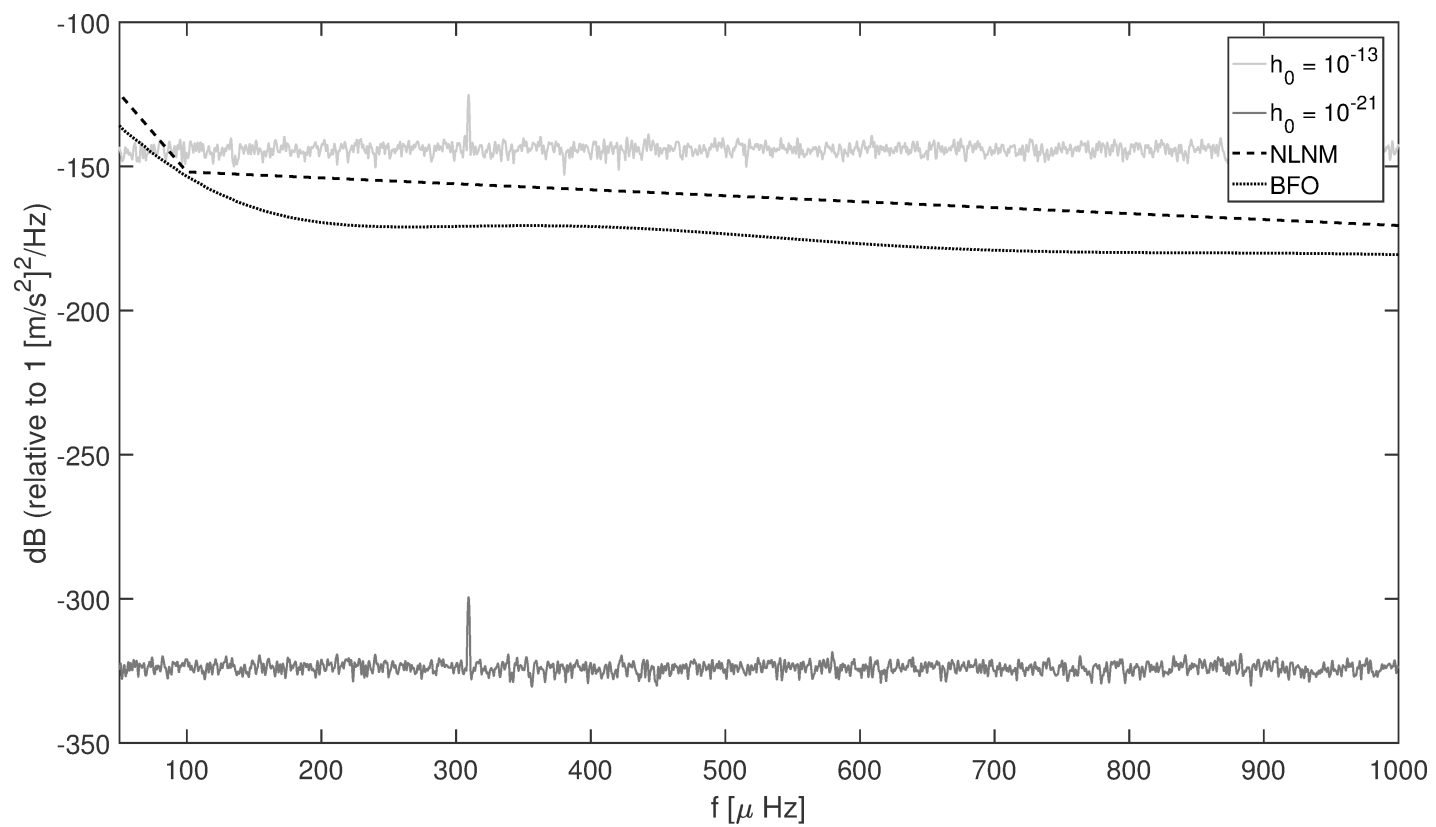

FIG. 3. Comparison in terms of power spectral densities between NLNM model, observed acceleration signal at the BFO (Germany) station and synthetic noisy signals $(52$ ) obtained by stacking 209 stations for ${ }_{0} \mathrm{~S}_{2}$ and $\mathrm{m}=-2$. Dark grey signal is obtained for $h_{0}=10^{-21}$ and with the standard deviation of injected white noise adjusted to allow for the signal to emerge from the noise. It was achieved with the rms value of NLNM model, at the frequency of the mode, but reduced by nine orders of magnitude. Light grey signal is obtained for the level of the noise set to match rms value of NLNM model at the frequency of the mode. The value of $h_{0}$ is increased until the signal emerges from the noise. That is achieved for $h_{0}=10^{-13}$.

In Fig. 3 we show the PSD of the signal (52) obtained by stacking 209 synthetic resonances computed at stations from seismometer and superconducting gravimeter networks for ${ }_{0} \mathrm{~S}_{2}$ and $m=-2$. Signal at each station depends on the station colatitude and longitude, GW amplitude which is set to $h_{0}=10^{-21}$ and is obtained for 60 days with $\Delta t=10 \mathrm{~s}$. The noise level is primarily set to the value estimated above, $\sigma_{N L N M}=8.0538 \cdot 10^{-9} \mathrm{~m} / \mathrm{s}^{2}$. Since, this high noise level completely prevails the signal, we start reducing the noise till our signal emerges from the noise. In the mentioned configuration the emerged signal is reached with $\sigma_{N L N M}=8.0538 \cdot 10^{-18} \mathrm{~m} / \mathrm{s}^{2}$, nine order of magnitude smaller than the first set. The stacking was performed with the optimal sequence estimation [54], based on the assumption that displacement on the Earth's surface is decomposed in spherical harmonics. On the same figure we plot the PSD value for the NLNM model and PSD for the Black 
Forest Observatory station, the quietest station [55]. It is clear that the GW signal is far below the detection level, which is already stated in the paragraph above. In the next example, showed on the same figure, we calculate the stacked signal with the noise standard deviation $\sigma_{N L N M}=8.0538 \cdot 10^{-9} \mathrm{~m} / \mathrm{s}^{2}$, and we increase GW source amplitude $h_{0}$ until the signal emerge from the noise. This is finally reached with $h_{0}=10^{-13}$ for 60 day long signals. This estimate achieved by stacking a large number of time-series is 3 orders of magnitude smaller than the one estimated in the previous paragraph. However this is still 5 orders of magnitude bigger than the expected GW source amplitudes in the $\mathrm{mHz}$ band $\left(\approx 10^{-18}\right)$ for possible astrophysical sources [9].

Besides NLNM, there is a more recent noise model proposed by [56] (further BLNM), where 118 Global Seismograph Network stations were analyzed in the duration of one year. The analysis was performed on vertical and horizontal components and data were not scrutinized for earthquakes and transients or any other variation. As such, the envelope of the first percentile of the empirical distribution for all station and channels is lower than NLNM for frequencies $<2.5 \mathrm{~Hz}$. The noise standard deviation for BLNM with sampling period of $10 \mathrm{~s}$ and vertical component is $\sigma_{B L N M}=6.92 \cdot 10^{-10} \mathrm{~m} / \mathrm{s}^{2}(-173.2 \mathrm{~dB})$. The conclusion above is similar, since the improvement by one order of magnitude for $\sigma_{N L N M}$, is not significantly sufficient. For horizontal displacement the standard deviation for $T_{0}=10 \mathrm{~s}$ is one order smaller, being $\sigma_{B L N M}=6.10 \cdot 10^{-9} \mathrm{~m} / \mathrm{s}^{2}(-154.3 \mathrm{~dB})$. This means that for 10 years one would need GW amplitude to be larger than $h_{0}>10^{-13}$ to be detected on Earth. There are two more noise models, one proposed by [57] and the other by [58, however they do not consider the lowest normal mode frequency band and therefore they could not be considered in this study.

From his flat-Earth model filled with a uniform isotropic elastic medium in the $1-\mathrm{Hz}$ frequency band Dyson [17] calculated the horizontal displacement for a horizontally incident GW to be $2 \cdot 10^{-17} \mathrm{~cm}=2 \cdot 10^{-10} \mathrm{~nm}$, which was the same result derived by Weber [59] and Dozmorov [30]. This value is five orders of magnitude smaller than ours, which is not surprising since Dyson pointed out that his estimation might be pessimistic because this estimate involved several assumptions that could be wrong by several orders of magnitude, such as the type of source, the absence of reflection or resonance effects in the seismic response. Recalculating Dyson's values, Sabbata et. al. [18] arrived at $1.4 \cdot 10^{-12} \mathrm{~cm}$ $=1.4 \cdot 10^{-5} \mathrm{~nm}$ for the peak displacement, just by considering different values for the incoming 
GW flux and Q-factor, and these values are already comparable with (55). Several studies search for seismic signals at pulsar frequencies but without success [19, 60, 61]. General conclusions were that the detection of such small signals is limited by Earth intrinsic noise and the short data series [62].

Many studies also provided the upper limits on the characteristic strain, the spectral energy density or dimensionless energy density of the GW stochastic backgrounds or GW burst [12, 13, 31, 33, 63]. The level of the stochastic gravitational radiation is conventionally expressed as the energy density relative to the critical energy density, that is the energy density per logarithmic frequency interval, or as a characteristic rms strain [47]. The most recent relevant estimation of the upper limit in the $\mathrm{mHz}$ band used an analytical solution of the Earth's normal modes for PREM model to calibrate normal mode amplitudes into GW strain data [25]. They estimated the upper limit of the GW energy density in the $0.035-0.15$ $\mathrm{Hz}$ frequency band, normalized by the critical energy density of the Universe, to be between $\Omega_{\mathrm{GW}}\left({ }_{0} \mathrm{~S}_{2}\right)=0.039$ to $\Omega_{\mathrm{GW}}\left({ }_{13} \mathrm{~S}_{2}\right)=0.12$. These values translated into strain amplitude spectral density lie between $h_{G W}\left({ }_{0} \mathrm{~S}_{2}\right)=2.2 \cdot 10^{-14} \mathrm{~Hz}^{1 / 2}$ to $h_{G W}\left({ }_{13} \mathrm{~S}_{2}\right)=6.2 \cdot 10^{-16} \mathrm{~Hz}^{1 / 2}$. The authors stated this is still by a large amount above the predicted levels of the GW background from the cosmological models.

In our example above we focus on the resonance effect between GW and only one normal mode ${ }_{0} \mathrm{~S}_{2}$ by setting $\omega_{g}={ }_{0} \omega_{2}$, however our results show that all the normal modes with the angular order $l=2$ should be excited by the GW. To understand the relationship between the resonance effect between different modes we calculate the resonance response for hundred normal modes $(n=0, \ldots, 99)$ of angular order $l=2$ (1 fundamental mode and 99 harmonics). The result, shown in Fig. 4, is calculated at the BFO station $\left(43.33^{\circ}, 8.33^{\circ}\right)$ for 30 day long time series with a constant $h_{0}$ set to 1 (for better visual clarity). We can see that some modes have higher resonance amplitudes than others, which makes them better candidates for the detection. To calculate the response of the Earth to the GW more precisely, one should consider the off-resonance modes as well. That is the full excitation response should consider the sum of the resonance and the off-resonance normal modes [33]. It is expected that during the resonance between GW and a normal mode, the contribution of the mode in resonance is the largest contribution (due to the source-time function). However, this is true for the low frequencies modes, while the largest contribution for the high frequency modes is not necessarily coming from the mode in the resonance [33]. To demonstrate 
a relation between resonance and the off-resonance effect we plot in Fig. 5 the absolute amplitude values versus the radial order $n$. Each row on the $y$-axis represents the radial order for which the resonance was calculated, therefore $\omega_{g}={ }_{n} \omega_{2}$, and each column on the $x$-axis represents the absolute amplitude value of the radial order in the off-resonance regime. What we would expect is to have the largest value in the diagonal of this square, which would indicate that the largest contribution comes form the mode in resonance. For example, from the figure, we can conclude that the first row on the $y$-axis stands for the values when $\omega_{g}={ }_{0} \omega_{2} x$ and in that case the largest contribution comes from ${ }_{0} \mathrm{~S}_{2}$. Unlike the case before, for the row six on the $y$-axis, the largest contribution comes from column $n=7$ on the $x$-axis and not $n=6$, what is expected. This tells us that the prevailing factor in the amplitude is not the resonant frequency anymore, but other factors such as ${ }_{n} \alpha$. Therefore when calculating the response of the Earth to the incoming GW it is more correct to include both the resonance and off-resonance effects. In reality it is hardly possible that the GW would have the exact frequency of the normal mode, consequently the full off-resonance effect should be considered. Thenceforth, the response should definitely consist of the sum of the normal modes closest to the GW frequency (Fig. 5) as well as other modes. 


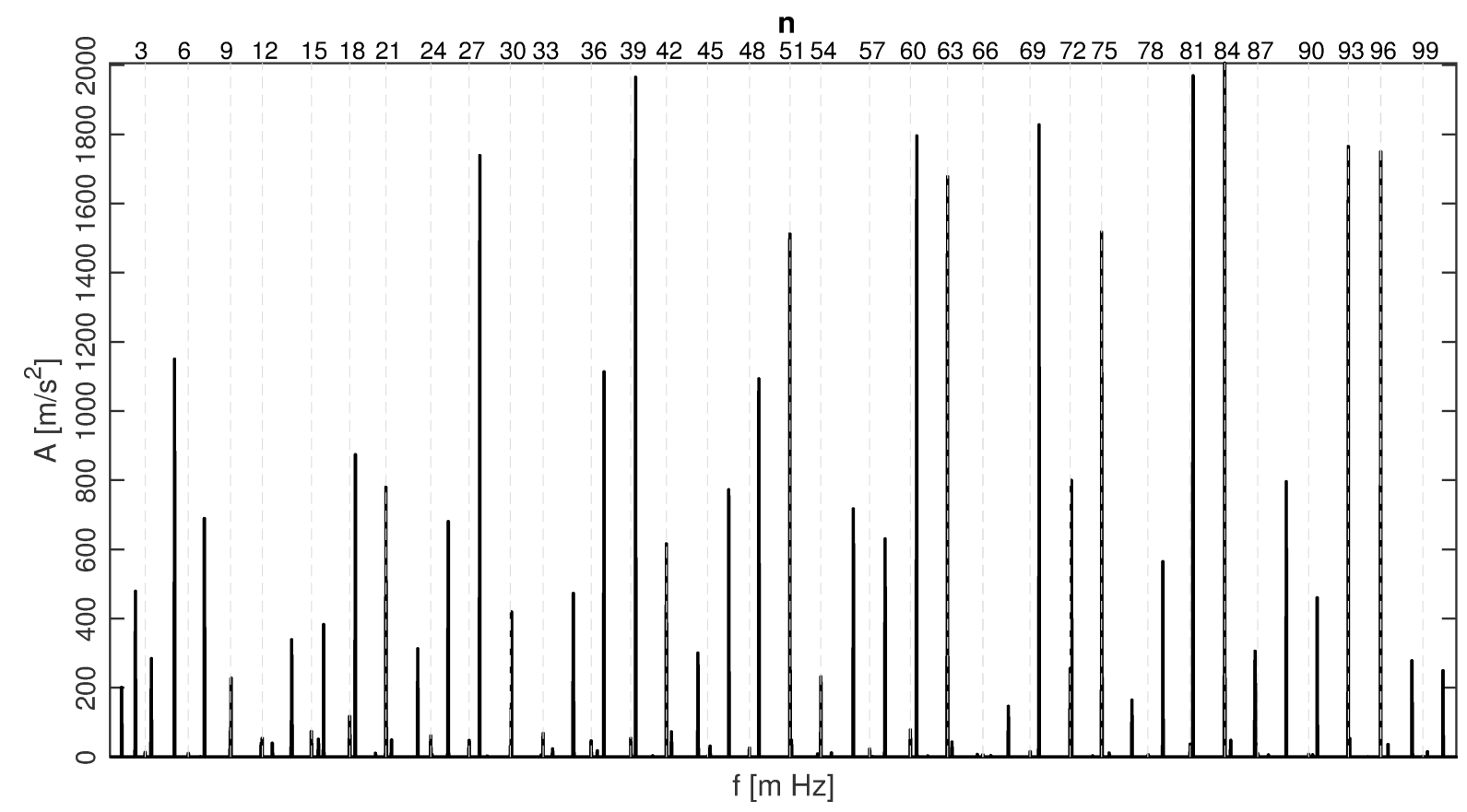

FIG. 4. Resonance excitation of the Earth's normal modes due to monochromatic GW passing through Earth. Each frequency spike represents a resonance for a different radial order of a quadrupole mode. The light grey lines represent the normal mode frequency as a function of the radial order $n$ indicated above the figure. 


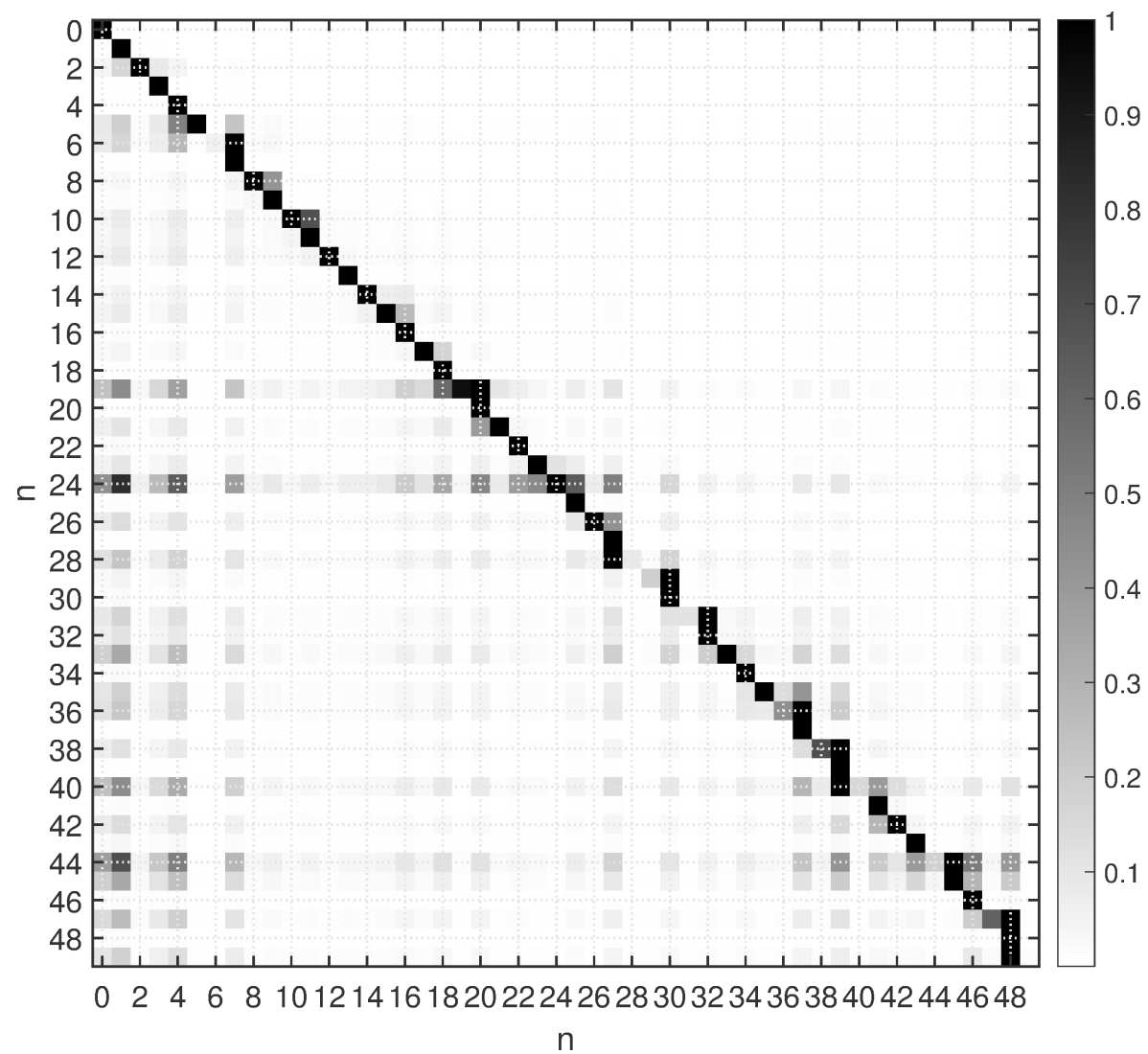

FIG. 5. The absolute amplitude values for resonance and off-resonance modes. Each row on $y$-axis labelled by $n$ represents the radial order of ${ }_{n} \mathrm{~S}_{2}$ mode in resonance, for which $\omega_{g}={ }_{0} \omega_{2}$, while each column on $x$-axis also labelled by $n$ represents modes that are in the off-resonance regime. Each row is normalized with the largest value in that row. The black color represent the normal mode with the largest contribution.

Theoretically, Earth resembles a spherical resonant-mass detector. It is argued that performances of the spherical resonant-mass detectors could improve the detection of GWs as compared to the resonant bars [47, 64, 65]. Firstly, due to their bigger mass they have a larger cross section for the absorption of GWs and hence a better sensitivity. Secondly, a sphere does not have a preferable orientation and offers a full sky coverage, unlike other detectors which have blind directions. Thirdly, using the information about the excited quadrupolar modes it is possible to reconstruct the arrival direction and polarization of GW. Weber [13] gave an estimate of the Earth cross section to be $10^{4} \mathrm{~m}^{2}$. Ruffini and Wheeler [66] estimated the resonance integral of the absorption cross section for radiation incident from random directions and with random polarizations for a fluid globe model. This 
globe, held in a shape of a sphere by only gravitational forces, has a uniform density with an average value of $5.517 \mathrm{~g} / \mathrm{cm}^{3}$ and thus the quadrupole vibration period of $94 \mathrm{~min}$. To roughly estimate the resonance integral for the Earth model with a quadrupolar vibration period of $54 \mathrm{~min}$, they adjusted the moment tensor for the two models and arrived to an absorption cross section value of $4.7 \mathrm{~cm}^{2} \mathrm{~Hz}$. By comparison, the cross-section of the spherical resonantmass detector, with the diameter of $3.2 \mathrm{~m}$, mass of $4.6 \cdot 10^{4} \mathrm{~kg}$ and frequency of $840 \mathrm{~Hz}$, has a resonance absorption cross section of $8.7 \cdot 10^{-20} \mathrm{~cm}^{2} \mathrm{~Hz}$ [64], which is much smaller than the Earth's cross-section. Furthermore, all spherical resonant-mass detectors have relatively small sizes and thus are suitable for the exploration of high frequencies regions, unlike Earth where we could explore the $\mathrm{mHz}$ frequency band.

The detection of elusive signals, such as GW, is a problem consisting of several parts. It depends on the instrument precision, the ubiquitous environmental noise, the modeling of the signal we want to find and techniques performed for the search. Considering the specific problem in this study, that is detecting the GWs using the resonance effect between GWs and the normal modes of Earth in the gravimetric and seismic data, not all categories aforementioned have been fully scrutinized. What we know, from the geophysical perspective, is that the instruments with the best sensitivity in the $0.3-1 \mathrm{mHz}$ band are superconducting gravimeters [55, 67], whose nominal sensitivity is generally referred to as $1 \mathrm{nGal}=10^{-11} \mathrm{~m} / \mathrm{s}^{2}$ [68]. Also, it has been shown that instrumental self-noise is not the main issue in the detection threshold, but the environmental noise and many geophysical processes, such as seismic, atmospheric and tidal perturbations, that have not been reduced from gravimetric and seismic data [52]. This problem is substantial and complex, since it is more difficult to control and model unknown geophysical processes than to just compensate them, such as in the case of the laser interferometrical free mass antenna procedure. For this purpose it would be really interesting to use seismic noise compensation in the laser interferometry for the study of geophysical processes that produce it [62]. Therefore, it is only reasonable to claim that to this day we are still only able to measure existing geophysical effects and estimate a new upper limit on the GW. One recent study [46] supported the idea of the whole Earth as a detector of the GW by utilizing the network of thousand of digital seismometers as a single gravitational antenna. They showed that in the frequency range $0.1-10 \mathrm{~Hz}$ it is possible to resolve absolute strains $h \lesssim 10^{-17}$ on burst gravitational pulses and $h \lesssim 10^{-21}$ on periodic signals. However this does not comply with theoretical 
predictions for the cosmic gravitational radiations.

\section{CONCLUSION}

In this paper we revisited the theoretical equations describing the interaction between Earth and GWs of astrophysical origin. This modeling is roughly based on several hypotheses. Firstly, GWs are monochromatic waves described by a source scalar value, a polarization tensor and a propagating vector. Secondly, the Earth is a non-rotating and anelastic body. Thirdly, the set-up is in the Earth's reference system. Fourthly, GWs are considered as a trigger of the Earth's normal modes, therefore they are represented as a force term in the Green tensor formalism. The derivation and analysis have shown that due to the fact that the GW tensor $h_{\mu \nu}$ is traceless and symmetric the only normal modes that couple with the GW are the ones with the degree $l=2$. Also, the spheroidal induced displacement depends on the source scalar value $h_{0}$, the displacement eigenfunction $\mathbf{s}_{k}$ and a constant $\alpha_{k}$, both depending on the Earth model, the source-time function $\bar{g}(t)$ and the three angles function $f^{m}(e, \lambda, \nu)$ defining the incoming $\mathrm{GW}$ in the Earth reference system. Specific configuration of GW angles $f^{m}(e, \lambda, \nu)$ triggers specific singlets within the ${ }_{n} \mathrm{~S}_{2}$ multiplet, and thus having information about individual singlets is giving us information about the position of the GW source in the sky. Considering the comparison between the resonant and off-resonant modes of low and high frequencies, it is shown that the low-frequency resonant modes have a larger response than the high-frequency modes and clearly the contributions of the low-frequency modes could be used exclusively in the computation of the induced displacement. However, it seems more reasonable to always consider the sum of all off-resonant modes near the frequency of the incoming GW to have a more representative solution.

Considering the measurements of the gravitationaly triggered normal modes, published values and the one in this study, show us that we are still obscured by the seismic noise of geophysical origin. Even though instruments with appropriate sensitivity may exist, envi-

ronmental noise will be a limiting factor. With the new era approaching new instruments of low-frequency sensitivity are developed, among others superconducting gravity gradiometers (SGG) [69] and atom interferometers [70, 171]. These instruments have promising standpoint with improved sensitivities over their predecessors. However, the problem of the unknown geophysical processes present in data still remains. 
The modelled interaction between Earth and GWs is restrained by some basic assumptions and can be improved. Those upgrades concern the fact that if we want to scrutinize more realistic GW sources we should not be restrained by the Earth reference system. Therefore, it is in our future interest to study the transformation from the celestial star systems to the terrestrial reference system and how this is affecting our final induced spheroidal displacement. This introduces some effects that we have ignored, like Earth's rotation and its associated effects, which may become important.

\section{ACKNOWLEDGMENTS}

This work was supported by the Programme National de Planétologie (PNP) of CNRS/INSU, co-funded by CNES. J. M. acknowledges also support from IPGS/UMR7516. We thank Sophie Lambotte for providing her code for calculating displacement time series for an anisotropic PREM Earth model. We are grateful to an anonymous reviewer for helpful comments that greatly improved manuscript.

\section{APPENDIX}

Comparing the solutions from [23] with ours is not completely straightforward because we do not use the same formalism. The dissimilarities are the topic of this Appendix. Also, when we rederive the solutions of [23] we find some inconsistencies that we are going to describe. In the following, the equations from [23] are inserted in square brackets.

Ben-Menahem stated that he derived the induced spheroidal field by using his equations [28], [35] and [36]. For the sake of comparison we assume that those equations are correct and we perform the same calculations. We also use his notation. The result is

$$
{ }_{n} \boldsymbol{u}_{m 2}(\boldsymbol{r}, t)=h_{0} \bar{g}_{n}(t)_{n} \boldsymbol{Q}_{m 2}^{*}(\boldsymbol{r})_{n} \wedge_{m 2}^{-1} \frac{8 \pi}{5 \sqrt{6}} f_{m}\left[a^{2} \mu(a)\left(y_{1 n}+3 y_{3 n}\right)-\int_{0}^{a} \frac{\partial \mu}{\partial r}\left(y_{1 n}+3 y_{3 n}\right) r^{2} d r\right],
$$


with function $f_{m}$ being

$$
\begin{aligned}
f_{m} & =\frac{\sqrt{6}}{4} \sin ^{2}(e) \mathrm{e}^{2 i \nu} \delta_{m, 0} \\
& +\cos ^{4}\left(\frac{e}{2}\right) \mathrm{e}^{2 i \lambda} \mathrm{e}^{2 i \nu} \delta_{m, 2} \\
& +\sin ^{4}\left(\frac{e}{2}\right) \mathrm{e}^{-2 i \lambda} \mathrm{e}^{2 i \nu} \delta_{m,-2} \\
& -2 \sin \left(\frac{e}{2}\right) \cos ^{3}\left(\frac{e}{2}\right) \mathrm{e}^{i \lambda} \mathrm{e}^{2 i \nu} \delta_{m, 1} \\
& -2 \sin ^{3}\left(\frac{e}{2}\right) \cos \left(\frac{e}{2}\right) \mathrm{e}^{-i \lambda} \mathrm{e}^{2 i \nu} \delta_{m,-1}
\end{aligned}
$$

Substituting the normalization factor defined as

$$
{ }_{n} \wedge_{m 2}=\frac{4 \pi}{5} \int_{0}^{a}\left(y_{1 n}^{2}+6 y_{3 n}^{2}\right) \rho(r) r^{2} d r
$$

into A.1 we arrive at the expression

$$
{ }_{n} \boldsymbol{u}_{m 2}(\boldsymbol{r}, t)=\frac{2}{\sqrt{6}} h_{0} \bar{g}_{n}(t)_{n} \boldsymbol{Q}_{m 2}^{*}(\boldsymbol{r}) f_{m} \tilde{\alpha}
$$

where we introduce the abbreviation defined as

$$
\tilde{\alpha}=\frac{a^{2} \mu(a)\left(y_{1 n}+3 y_{3 n}\right)-\int_{0}^{a} \frac{\partial \mu}{\partial r}\left(y_{1 n}+3 y_{3 n}\right) r^{2} d r}{\int_{0}^{a}\left(y_{1 n}^{2}+6 y_{3 n}^{2}\right) \rho(r) r^{2} d r} .
$$

This can be compared with his solution [45]

$$
{ }_{n} \boldsymbol{u}_{m 2}(\boldsymbol{r}, t)^{\mathrm{BM}}=(-1)^{m} \frac{2}{\sqrt{6}} h_{0} \bar{g}_{n}(t) \tilde{\alpha} \Re\left\{{ }_{n} \boldsymbol{Q}_{m 2}(\boldsymbol{r}) \sin ^{2-m}\left(\frac{e}{2}\right) \cos ^{2+m}\left(\frac{e}{2}\right) \mathrm{e}^{i m(\lambda+\nu)}\right\},
$$

which can be recast as

$$
{ }_{n} \boldsymbol{u}_{m 2}(\boldsymbol{r}, t)^{\mathrm{BM}}=\frac{2}{\sqrt{6}} h_{0} \bar{g}_{n}(t){ }_{n} \boldsymbol{Q}_{m 2}(\boldsymbol{r}) f_{m}^{\mathrm{BM}} \tilde{\alpha}
$$

with the function $f_{m}^{\mathrm{BM}}$ defined by

$$
\begin{aligned}
f_{m}^{\mathrm{BM}}= & =\frac{1}{4} \sin ^{2}(e) \mathrm{e}^{2 i \nu} \delta_{m, 0} \\
& +\cos ^{4}\left(\frac{e}{2}\right) \mathrm{e}^{2 i \lambda} \mathrm{e}^{2 i \nu} \delta_{m, 2} \\
& +\sin ^{4}\left(\frac{e}{2}\right) \mathrm{e}^{-2 i \lambda} \mathrm{e}^{2 i \nu} \delta_{m,-2} \\
& -\sin \left(\frac{e}{2}\right) \cos ^{3}\left(\frac{e}{2}\right) \mathrm{e}^{i \lambda} \mathrm{e}^{2 i \nu} \delta_{m, 1} \\
& -\sin ^{3}\left(\frac{e}{2}\right) \cos \left(\frac{e}{2}\right) \mathrm{e}^{-i \lambda} \mathrm{e}^{2 i \nu} \delta_{m,-1}
\end{aligned}
$$


Just by simple comparison of the expressions (A.4) and (A.7) there are two differences: firstly, Ben-Menahem did not include the complex conjugate for the displacement eigenfunction ${ }_{n} \boldsymbol{Q}_{m 2}(\boldsymbol{r})$, even though it exists in the definition given by relation [26]; secondly, he misplaced the coefficients $\theta_{m}$ defined in his expression [36], since these are the values missing in expression (A.8) compared to A.2.

If we try a different approach and recalculate the induced response [45] using expressions [42] and [43] obtained by contracting polarization tensor [15] and quadrupole moment tensor [44] we should obtain the same result [45]. Thus, we have

$$
\begin{aligned}
F_{S_{1}} & =\frac{a^{2} \mu(a) y_{1 n}-\int_{0}^{a} \frac{\partial \mu}{\partial r} y_{1 n} r^{2} d r}{3 \int_{0}^{a} \rho_{0} y_{3 n} r^{3} d r}\left(\varepsilon \varepsilon: \delta D_{B}\right) \\
& =\left(a^{2} \mu(a) y_{1 n}-\int_{0}^{a} \frac{\partial \mu}{\partial r} y_{1 n} r^{2} d r\right) \frac{1}{3} \frac{24 \pi}{5 \sqrt{6}} \delta_{l, 2} \frac{1}{2} 4 \\
& {\left[\frac{\sqrt{6}}{4} \sin ^{2} e \mathrm{e}^{2 i \nu} \delta_{m, 0}\right.} \\
& +\cos ^{4}\left(\frac{e}{2}\right) \mathrm{e}^{2 i \lambda} \mathrm{e}^{2 i \nu} \delta_{m, 2} \\
& +\sin ^{4}\left(\frac{e}{2}\right) \mathrm{e}^{-2 i \lambda} \mathrm{e}^{2 i \nu} \delta_{m,-2} \\
& +2 \sin ^{3}\left(\frac{e}{2}\right) \cos \left(\frac{e}{2}\right) \mathrm{e}^{-i \lambda} \mathrm{e}^{2 i \nu} \delta_{m, 1} \\
& \left.-2 \sin \left(\frac{e}{2}\right) \cos ^{3}\left(\frac{e}{2}\right) \mathrm{e}^{i \lambda} \mathrm{e}^{2 i \nu} \delta_{m,-1}\right] \\
& =\frac{16 \pi}{5 \sqrt{6}} \delta_{l, 2} f^{m ; \mathrm{BM} 2}(e, \lambda, \nu)\left(a^{2} \mu(a) y_{1 n}-\int_{0}^{a} \frac{\partial \mu}{\partial r} y_{1 n} r^{2} d r\right)
\end{aligned}
$$

with function $f^{m ; \mathrm{BM} 2}$ defined as

$$
\begin{aligned}
f^{m ; \mathrm{BM} 2}(e, \lambda, \nu) & =\frac{\sqrt{6}}{4} \sin ^{2} e \mathrm{e}^{2 i \nu} \delta_{m, 0} \\
& +\cos ^{4}\left(\frac{e}{2}\right) \mathrm{e}^{2 i \lambda} \mathrm{e}^{2 i \nu} \delta_{m, 2} \\
& +\sin ^{4}\left(\frac{e}{2}\right) \mathrm{e}^{-2 i \lambda} \mathrm{e}^{2 i \nu} \delta_{m,-2} \\
& +2 \sin ^{3}\left(\frac{e}{2}\right) \cos \left(\frac{e}{2}\right) \mathrm{e}^{-i \lambda} \mathrm{e}^{2 i \nu} \delta_{m, 1} \\
& -2 \sin \left(\frac{e}{2}\right) \cos ^{3}\left(\frac{e}{2}\right) \mathrm{e}^{i \lambda} \mathrm{e}^{2 i \nu} \delta_{m,-1}
\end{aligned}
$$

Substituting this in [28] we have

$$
{ }_{n} \boldsymbol{u}_{m 2}(\boldsymbol{r}, t)^{\mathrm{BM} 2}=\frac{4}{\sqrt{6}} h_{0} \bar{g}_{n}(t){ }_{n} \boldsymbol{Q}_{m 2}(\boldsymbol{r}) f_{m}^{\mathrm{BM} 2} \tilde{\alpha}
$$


Eventually, we see that this approach also yields some differences. Function A.10 is more similar to the relation A.2 than A.8. However, the values multiplying the Kronecker symbols $\delta_{m, 1}$ and $\delta_{m,-1}$ are inverted compared to A.2 and A.8. We highly suspect that the reason for this comes from the definition of the quadrupole moment tensor [44], which we were not able to reproduce.

Let us now derive the solution of this study using fully normalized complex spherical harmonics (unlike the real spherical harmonics that we used in the main body of the text) defined as

$$
Y_{l m}(\theta, \phi)=\sqrt{\frac{2 l+1}{4 \pi}} \sqrt{\frac{(l-m) !}{(l+m) !}} P_{l m}(\cos \theta) \mathrm{e}^{i m \phi},
$$

with the associated Legendre function $P_{l m}(x)$ defined by

$$
P_{l m}(x)=\frac{(-1)^{m}}{2^{l} l !}\left(1-x^{2}\right)^{m / 2} \frac{d^{l+m}}{d x^{l+m}}\left(x^{2}-1\right)^{l} .
$$

This assumption yields for the integrals $I_{1}$ and $I_{2}$ solutions that are different from our results 29) and (32). They are

$$
\begin{aligned}
I_{1} & =\frac{2 \sqrt{\pi}}{3} \delta_{l, 0} \delta_{m, 0}\left[\begin{array}{lll}
1 & 0 & 0 \\
0 & 1 & 0 \\
0 & 0 & 1
\end{array}\right]+\frac{2}{3} \sqrt{\frac{\pi}{5}} \delta_{l, 2} \delta_{m, 0}\left[\begin{array}{ccc}
-1 & 0 & 0 \\
0 & -1 & 0 \\
0 & 0 & 2
\end{array}\right] \\
& +\sqrt{\frac{2 \pi}{15}} \delta_{l, 2}\left[\begin{array}{ccc}
\delta_{m, 2}+\delta_{m,-2} & i \delta_{m, 2}-i \delta_{m,-2} & -\delta_{m, 1}-\delta_{m,-1} \\
i \delta_{m, 2}-i \delta_{m,-2} & -\delta_{m, 2}-\delta_{m,-2} & -i \delta_{m, 1}+-i \delta_{m,-1} \\
\delta_{m, 1}-\delta_{m,-1} & -i \delta_{m, 1}-i \delta_{m,-1} & 0
\end{array}\right]
\end{aligned}
$$

and

$$
\begin{aligned}
I_{2} & =2 \sqrt{\frac{\pi}{5}} \delta_{l, 2} \delta_{m, 0}\left[\begin{array}{ccc}
-1 & 0 & 0 \\
0 & -1 & 0 \\
0 & 0 & 2
\end{array}\right] \\
& +3 \sqrt{\frac{2 \pi}{15}} \delta_{l, 2}\left[\begin{array}{ccc}
\delta_{m, 2}+\delta_{m,-2} & i \delta_{m, 2}-i \delta_{m,-2} & -\delta_{m, 1}-\delta_{m,-1} \\
i \delta_{m, 2}-i \delta_{m,-2} & -\delta_{m, 2}-\delta_{m,-2} & -i \delta_{m, 1}+-i \delta_{m,-1} \\
\delta_{m, 1}-\delta_{m,-1} & -i \delta_{m, 1}-i \delta_{m,-1} & 0
\end{array}\right]
\end{aligned}
$$

This, finally, gives us a new induced spheroidal response

$$
\mathbf{s}_{k}(\mathbf{r}, t)^{\mathrm{CSH}}=2 \sqrt{\frac{2 \pi}{15}} h_{0} \mathbf{s}_{k}(\mathbf{r}) \bar{g}(t) \delta_{l, 2} f_{b}^{m ; \mathrm{CSH}}(e, \lambda, \nu) \alpha_{k}
$$


with function $f_{b}^{m ; \mathrm{CSH}}(e, \lambda, \nu)$ defined as

$$
\begin{aligned}
f_{b}^{m ; \operatorname{CSH}}(e, \lambda, \nu) & =\frac{\sqrt{6}}{4} \sin ^{2} e \mathrm{e}^{2 i \nu} \delta_{m, 0} \\
& +\cos ^{4}\left(\frac{e}{2}\right) \mathrm{e}^{2 i \lambda} \mathrm{e}^{2 i \nu} \delta_{m, 2} \\
& +\sin ^{4}\left(\frac{e}{2}\right) \mathrm{e}^{-2 i \lambda} \mathrm{e}^{2 i \nu} \delta_{m,-2} \\
& +2 \sin \left(\frac{e}{2}\right) \cos ^{3}\left(\frac{e}{2}\right) \mathrm{e}^{i \lambda} \mathrm{e}^{2 i \nu} \delta_{m, 1} . \\
& +2 \sin ^{3}\left(\frac{e}{2}\right) \cos \left(\frac{e}{2}\right) \mathrm{e}^{-i \lambda} \mathrm{e}^{2 i \nu} \delta_{m,-1},
\end{aligned}
$$

where CSH stand for complex spherical harmonic and $\alpha_{k}$ for the model dependent function (36). We repeat the same calculation just with the spherical harmonics defined as

$$
\tilde{Y}_{l m}=(-1)^{m} \sqrt{\frac{2 l+1}{4 \pi}} Y_{l m}
$$

which gives us

$$
\tilde{Y}_{l m}=\left(\frac{2 l+1}{4 \pi}\right) \sqrt{\frac{(l-m) !}{(l+m) !}} \tilde{P}_{l m}(\cos \theta) \mathrm{e}^{i m \phi},
$$

where for the associated Legendre function $\tilde{P}_{l m}(x)$ the valid relation is

$$
P_{l m}(x)=(-1)^{m} \tilde{P}_{l m}(x) .
$$

This definition of spherical harmonics corresponds to the one in [48] that is presumably used in [23]. The induced spheroidal response becomes

$$
\mathbf{s}_{k}(\mathbf{r}, t)^{\mathrm{CSH} 2}=\frac{2}{\sqrt{6}} h_{0} \mathbf{s}_{k}(\mathbf{r}) \bar{g}(t) \delta_{l, 2} \tilde{f}^{m ; \mathrm{CSH} 2}(e, \lambda, \nu) \alpha_{k},
$$

with

$$
\begin{aligned}
\tilde{f}^{m ; \operatorname{CSH} 2}(e, \lambda, \nu) & =\frac{\sqrt{6}}{4} \sin ^{2} e \mathrm{e}^{2 i \nu} \delta_{m, 0} \\
& +\cos ^{4}\left(\frac{e}{2}\right) \mathrm{e}^{2 i \lambda} \mathrm{e}^{2 i \nu} \delta_{m, 2} \\
& +\sin ^{4}\left(\frac{e}{2}\right) \mathrm{e}^{-2 i \lambda} \mathrm{e}^{2 i \nu} \delta_{m,-2} \\
& -2 \sin \left(\frac{e}{2}\right) \cos ^{3}\left(\frac{e}{2}\right) \mathrm{e}^{i \lambda} \mathrm{e}^{2 i \nu} \delta_{m, 1} . \\
& -2 \sin ^{3}\left(\frac{e}{2}\right) \cos \left(\frac{e}{2}\right) \mathrm{e}^{-i \lambda} \mathrm{e}^{2 i \nu} \delta_{m,-1} .
\end{aligned}
$$

Therefore, one can use the transformation (A.18) between two definitions of spherical harmonics to obtain similar but not exactly the same relations. With this transformation we arrive at the same expression for A.2 and A.22, while relation A.21 still has some 
differences compared to relation (A.4). These dissimilarities concern how we define the displacement eigenfunctions and what normalization we use for the radial eigenfunctions. One of the differences concerns the definition of the Green tensor. We use a formalism where the definition of the Green tensor depends on the Earth model (see page 231 in [45]). In [48] those differences are not explicitly emphasised. In [23] Ben-Menahem stated that he developed the displacement for radially heterogeneous, anelastic self-gravitating, rotating Earth models and thus he used the Green tensor defined by relation [25], depending on the real radial eigenfunctions and complex spherical harmonics. Additionally, his displacement eigenfunction at the receiver is complex conjugate. For the same Earth model we would use Green tensors containing complex radial eigenfunctions and complex spherical harmonics [45]. Moreover, the displacement eigenfunction at the source would be dual. However, this could be simplified: anelasticity is ignored, therefore the radial eigenfunctions become real and the displacement eigenfunctions at the source become complex conjugate. At the end, we would have complex conjugate displacement eigenfunction at source unlike Ben-Menahem. Thus, if we follow our formalism for radially heterogeneous, anelastic self-gravitating, rotating Earth model we would derive integrals A.14 and A.15) for complex conjugate fully normalized spherical harmonic. In Tab. I we compare the functions that would be used in these two studies to derive displacement for radially heterogeneous, anelastic selfgravitating, rotating Earth model. In summary, the final solution highly depends on the spherical harmonic normalization, therefore on the normalization of radial eigenfunctions, on the definition of Green tensors and on the definition of the displacement eigenfunctions. 
TABLE I. List of functions (spherical harmonics, associated Legendre functions, Green tensors, displacement eigenfunctions, normalization of displacement eigenfunctions, respectively) that are used to develop displacement for radially heterogeneous, anelastic self-gravitating, rotating Earth model for Ben-Menahem (1983) and Dahlen \& Tromp (1998) formalism. Constants are $\Omega_{l m}=$ $\frac{4 \pi}{2 l+1} \frac{(l-m) !}{(l+m) !}$ and $\kappa=\sqrt{(l(l+1))}$. Normalization of the displacement eigenfunction for Dahlen \& Tromp (1998) is written for spherical non-rotating elastically isotropic Earth model, since the rotation is treated as a perturbation.

\begin{tabular}{ll}
\hline \hline Ben-Menahem $(1983)$ & This study \\
\hline$Y_{l m}(\theta, \phi)=\sqrt{\frac{(l-m) !}{(l+m) !}} P_{l m}(\cos \theta) \mathrm{e}^{i m \phi}$ & $Y_{l m}(\theta, \phi)=\sqrt{\frac{2 l+1}{4 \pi}} \sqrt{\frac{(l-m) !}{(l+m) !}} P_{l m}(\cos \theta) \mathrm{e}^{i m \phi}$ \\
$P_{l m}(\cos \theta)=\frac{1}{2^{l} l ! !}\left(1-\cos ^{2} \theta\right)^{m / 2} \frac{d^{l+m}}{\left.d \cos ^{l l+m}\right)}\left(\cos ^{2} \theta-1\right)^{l}$ & $P_{l m}(\cos \theta)=\frac{(-1)^{m}}{2^{l} l !}\left(1-\cos ^{2} \theta\right)^{m / 2} \frac{d^{l+m}}{d \cos ^{l l+m]}}\left(\cos ^{2} \theta-1\right)^{l}$ \\
$\mathbf{G}_{k}\left(\mathbf{r}, \mathbf{r}^{\prime} ; t\right)=\mathbf{Q}_{k}^{*}(\mathbf{r}) \mathbf{Q}_{k}\left(\mathbf{r}^{\prime}\right) \bar{g}(t) \wedge_{k}$ & $\mathbf{G}_{k}\left(\mathbf{r}, \mathbf{r}^{\prime} ; t\right)=\left(i \nu_{k}\right)^{-1} \mathbf{s}_{k}(\mathbf{r}) \mathbf{s}_{k}^{*}\left(\mathbf{r}^{\prime}\right) \mathrm{e}^{i \nu_{k} t}$ \\
$\mathbf{Q}_{k}(\mathbf{r})=y_{1 n}(r) \hat{\mathbf{e}}_{r} Y_{l m}(\theta, \phi)+y_{3 n}(r) \nabla_{1} Y_{l m}(\theta, \phi)$ & $\mathbf{s}_{k}(\mathbf{r})=U_{k}(r) \hat{\mathbf{e}}_{r} Y_{l m}(\theta, \phi)+\kappa^{-1} V_{k}(r) \nabla_{1} Y_{l m}(\theta, \phi)$ \\
$\int_{V} \mathbf{Q}_{k}(\mathbf{r}) \mathbf{Q}_{k}^{*}(\mathbf{r})(\mathbf{r}) \rho_{0}(r) d V=\Omega_{m l} I_{n}^{S}$ & $\int_{\otimes} \rho^{0} \mathbf{s}_{k} \cdot \mathbf{s}_{k}^{\prime} d V=\delta_{k k^{\prime}}$ \\
$I_{n}^{S}=\int_{0}^{a}\left(y_{1 n}^{2}+l(l+1) y_{3 n}^{2}\right) \rho(r) r^{2} d r$ & - \\
\hline \hline
\end{tabular}

[1] B. P. Abbott et al. (The LIGO Scientific Collaboration and The Virgo Collaboration), Physical Review Letter 116, 061102 (2016).

[2] A. Einstein, Preussische Akademie der Wissenschaften, Sitzungsberichte 1, 688 (1916).

[3] A. Einstein, Preussische Akademie der Wissenschaften, Sitzungsberichte 1, 154 (1918).

[4] B. P. Abbott et al. (The LIGO Scientific Collaboration and The Virgo Collaboration), (2018), arXiv:1811.12907.

[5] J. Aasi et al. (The LIGO Scientific Collaboration), Classical and Quantum Gravity 32 (2015).

[6] F. Acernese et al., Classical and Quantum Gravity 32 (2014).

[7] P. Amaro-Seoane et al., (2012), arXiv:1201.3621.

[8] R. S. Foster and D. C. Backer, Astrophysical Journal 361, 300 (1990).

[9] C. J. Moore, R. H. Cole, and C. P. L. Berry, Classical and Quantum Gravity 32 (2015).

[10] F. A. E. Pirani, General Relativity and Gravitation 41, 1215 (2009).

[11] J. Weber, Physical Review 117, 306 (1959).

[12] R. L. Forward, D. Zipoy, J. Weber, S. Smith, and H. Benioff, Nature 2, 573 (1961).

[13] J. Weber, Physical Review Letter 18, 498 (1967). 
[14] V. S. Tuman, Nature 230, 101 (1971).

[15] V. S. Tuman, General Relativity and Gravitation 4, 279 (1973).

[16] E. A. Flinn, Nature 232, 112 (1971).

[17] J. Dyson, F., The Astrophysical Journal 156, 529 (1969).

[18] V. De Sabbata, P. Fortini, C. Gualdi, and S. Petralia, Annals of Geophysics 23, 21 (1970).

[19] T. S. Mast, J. E. Nelson, and J. Saarloos, The Astrophysical Journal 187, L49 (1974).

[20] N. Ashby and J. Dreitlein, Physical Review D 12, 336 (1975).

[21] B. Linet, General Relativity and Gravitation 16, 89 (1984).

[22] O. G. Jensen, Reviews of geophysics and space physics 17, 2057 (1979).

[23] A. Ben-Menahem, II Nuovo Cimento C 6, 49 (1983).

[24] M. Coughlin and J. Harms, Physical review letters 112, 101102 (2014).

[25] M. Coughlin and J. Harms, Physical review D 90, 042005 (2014).

[26] M. Coughlin and J. Harms, Physical review D 90, 102001 (2014).

[27] B. Carter and H. Quintana, Proceedings of the Royal Society of London A 331, 57 (1972).

[28] E. N. Glass and J. Winicourt, Journal of Mathematical Physics 13, 1934 (1972).

[29] I. M. Dozmorov, Soviet Physics Journal 19, 741 (1976).

[30] I. M. Dozmorov, Soviet Physics Journal 19, 883 (1976).

[31] R. L. Zimmerman and R. W. Hellings, The Astrophysical Journal 241, 475 (1980).

[32] R. Walgate, Nature 305, 665 (1983).

[33] S. P. Boughn and J. R. Kuhn, The Astrophysical Journal 286, 387 (1984).

[34] H. G. Khosroshahi and Y. Sobouti, Astronomy \& Astrophysics 321, 1024 (1997).

[35] D. M. Siegel and M. Roth, Monthly Notices of the Royal Astronomical Society 408, 1742 (2010).

[36] D. M. Siegel and M. Roth, The Astrophysical Journal 729, 1 (2011).

[37] D. M. Siegel and M. Roth, Astronomische Nachrichten 333, 978 (2012).

[38] D. M. Siegel and M. Roth, The Astrophysical Journal 784, 1 (2014).

[39] I. Lopes and J. Silk, The Astrophysical Journal 794, 1 (2014).

[40] I. Lopes and J. Silk, The Astrophysical Journal 807, 1 (2015).

[41] I. Lopes and J. Silk, The Astrophysical Journal 844, 1 (2017).

[42] I. Lopes, Physical Review D 95, 123015 (2017).

[43] B. McKernan, K. E. S. Ford, B. Kocsis, and Z. Haiman, Monthly Notices of the Royal 
Astronomical Society: Letters 445, L74 (2014).

[44] T. L. Campante, I. Lopes, D. Bossini, A. Miglio1, and W. J. Chaplin, Astronomy in Focus , $363(2015)$.

[45] F. A. Dahlen and J. Tromp, Theoretical global seismology (Princeton University Press, 1998) p. 1025 .

[46] F. Mulargia and A. Kamenshchik, Physics Letters A 380, 1503 (2016).

[47] M. Maggiore, Gravitational Waves, Volume 1, Theory and Experiments (Oxford University Press, 2008) p. 554.

[48] A. Ben-Menahem and J. Singh, S., Seismic waves and sources (Mineola, Dover Publications, Inc., 1981) p. 1108.

[49] A. M. Dziewonski and D. L. Anderson, Physics of the Earth and Planetary Interiors 25, 297 (1981).

[50] J. H. Woodhouse, "In:Doornbos DJ. (ed) Seismological Algorithms, Computational Methods and Computer Programs," (London: Academic Press, 1988) Chap. The calculation of Eigenfrequencies and Eigenfunctions of the tree oscillations of the Earth and the Sun, pp. $321-370$.

[51] A. Ben-Menahem, M. Israel, and U. Levite, Geophysical Journal of the Royal Astronomical Society 25, 307 (1971).

[52] S. Rosat and J. Hinderer, Scientific Reports 8, 15324 (2018).

[53] J. Peterson, Open-File Report 93-332. U.S. Department of Interior, Geological Survey, Albuquerque, New Mexico (1993).

[54] H. Ding and W.-B. Shen, Journal of Geophysical Research: Solid Earth 118, 5018 (2013).

[55] S. Rosat and J. Hinderer, Bulletin of the Seismological Society of America 101, 1233 (2011).

[56] J. Berger, P. Davis, and Ekström, Journal of Geophysical Research 109, 1 (2004).

[57] D. E. McNamara and R. P. Buland, Bulletin of the Seismological Society of America 94, 1517 (2004).

[58] S. Castellaro and F. Mulargia, Seismological Research Letters 83, 39 (2012).

[59] J. Weber, Physical Review Letters 21, 395 (1968).

[60] R. A. Wiggins and F. Press, Journal of Geophysical Research 74, 5351 (1969).

[61] D. Sadeh and M. Meidav, Nature 240, 136 (1972).

[62] V. N. Rundeko, in Gravitational wave experiments, edited by E. Coccia, G. Pizzella, and 
F.Ronga (World Scientific Publishing Co. Pte. Ud., P 0 Box 128, Farrer Road, Singapore 9128, 1994) pp. 277-281.

[63] V. K. Kravchuk, A. V. Gusev, and V. N. Rudenko, international Journal of Modern Physics D 4, $63(1995)$.

[64] C. Z. Zhou and P. F. Michelson, Physical Review D 51 (1995).

[65] J. A. Lobo, Physical Review D 52, 591 (1995).

[66] R. Ruffini and J. A. Wheeler, "Relativistic cosmology and space platforms," in Proceedings of the Conference on Space Physics (European Space Research Organization, Paris, France, 1971).

[67] R. Widmer-Schnidrig, Bulletin of the Seismological Society of America 93, 1370 (2003).

[68] J. Hinderer, D. Crossley, and R. J. Warburton, Treatise Geophys. 3, 65 (2007).

[69] C. E. Griggs, M. V. Moody, R. S. Norton, H. J. Paik, and K. Venkateswara, Physical Review Applied 8, 064024 (2017).

[70] R. Geiger, L. Amand, A. Bertoldi, B. Canuel, W. Chaibi, C. Danquigny, I. Dutta, B. Fang, S. Gaffet, J. Gillot, D. Holleville, A. Landragin, M. Merzougui, I. Riou, D. Savoie, and P. Bouyer, (2015), arXiv:1505.07137.

[71] B. Canuel et al., Scientific Reports 8 (2018). 